\title{
Terrestrial Free-Space Optical Communications
}

\author{
Ghassemlooy, Z. and Popoola, W. O. \\ Optical Communications Research Group, NCRLab, \\ Northumbria University, Newcastle upon Tyne, UK
}

\section{Introduction}

Free-space optical communication (FSO) or better still laser communication is an age long technology that entails the transmission of information laden optical radiation through the atmosphere from one point to the other. The earliest form of FSO could be said to be the Alexander Graham Bell's Photophone of 1880. In his experiment, Bell modulated the Sun radiation with voice signal and transmitted it over a distance of about 200 metres. The receiver was made of a parabolic mirror with a selenium cell at its focal point. However, the experiment did not go very well because of the crudity of the devices used and the intermittent nature of the Sun radiation. The fortune of FSO changed in the 1960s with the discovery of optical sources, most importantly the laser. A flurry of FSO demonstrations was recorded in the early 1960s into 1970s. Some of these included the: spectacular transmission of television signal over a 30 mile $(48 \mathrm{~km})$ distance using GaAs light emitting diode by researchers working in the MIT Lincolns Laboratory in 1962, a record 118 miles $(190 \mathrm{~km})$ transmission of voice modulated He-Ne laser between Panamint Ridge and San Gabriel Mountain, USA in May 1963 and the first TV-over-laser demonstration in March 1963 by a group of researchers working in the North American Aviation. The first laser link to handle commercial traffic was built in Japan by Nippon Electric Company (NEC) around 1970. The link was a full duplex $0.6328 \mu \mathrm{m}$ He-Ne laser FSO between Yokohama and Tamagawa, a distance of $14 \mathrm{~km}$ (Goodwin, 1970).

From this time on, FSO has continued to be researched and used chiefly by the military for covert communications. FSO has also been heavily researched for deep space applications by NASA and ESA with programmes such as the then Mars Laser Communication Demonstration (MLCD) and the Semiconductor-laser Inter-satellite Link Experiment (SILEX) respectively. Although, deep space FSO lies outside the scope of our discussion here, it is worth mentioning that over the past decade, near Earth FSO were successfully demonstrated in space between satellites at data rates of up to $10 \mathrm{Gbps}$ (Hemmati, 2006). In spite of early knowledge of the necessary techniques to build an operational laser communication system, the usefulness and practicality of a laser communication system was until recently questionable for many reasons (Goodwin, 1970): First, existing communications systems were adequate to handle the demands of the time. Second, considerable research and development were required to improve the reliability of components to assure reliable system operation. Third, a system in the atmosphere would 
always be subject to interruption in the presence of heavy fog. Fourth, use of the system in space where atmospheric effects could be neglected required accurate pointing and tracking optical systems which were not then available. In view of these problems, it is not surprising that until now, FSO had to endure a slow penetration into the access network.

But with the rapid development and maturity of optoelectronic devices, FSO has now witnessed a re-birth. Also, the increasing demand for more bandwidth in the face of new and emerging applications implies that the old practice of relying on just one access technology to connect with the end users has to give way. These forces coupled with the recorded success of FSO in military applications have rejuvenated interest in its civil applications within the access network. Several successful field trials have been recorded in the last few years in various parts of the world which have further encouraged investments in the field. This has now culminated into the increased commercialisation and the deployment of FSO in today's communication infrastructures.

FSO has now emerged as a commercially viable alternative to radio frequency (RF) and millimetre wave wireless systems for reliable and rapid deployment of data and voice networks. RF and millimetre wave technologies wireless networks can offer data rates from tens of Mbps (point-to-multipoint) up to several hundred Mbps (point-to-point). However, there is a limitation to their market penetration due to spectrum congestion, licensing issues and interference from unlicensed bands. The future emerging license-free bands are promising, but still have certain bandwidth and range limitations compared to the FSO. The short-range FSO links are used as an alternative to the RF links for the last or first mile to provide broadband access network to businesses as well as a high bandwidth bridge between the local area networks (LANs), metropolitan area networks (MANs) and wide area networks (WANs) (Pelton, 1998).

Full duplex FSO systems running at up to $1.25 \mathrm{Gbps}$ between two static nodes and covering a range of over $4 \mathrm{~km}$ in clear weather conditions are now common sights in today's market. Integrated FSO/fibre communication systems and wavelength division multiplexed (WDM) FSO systems are currently at experimental stages and not yet deployed in the market. One of such demonstrations is the single-mode fibre integrated $10 \mathrm{Gbps}$ WDM FSO carried out in Japan (Kazaura et al., 2007). The earlier scepticism about FSO's efficacy, its dwindling acceptability by service providers and slow market penetration that bedevilled it in the 1980s are now rapidly fading away judging by the number of service providers, organisations, government and private establishments that now incorporate FSO into their network infrastructure. Terrestrial FSO has now proven to be a viable complementary technology in addressing the contemporary communication challenges; most especially the bandwidth/high data rate requirements of end users at an affordable cost. The fact that FSO is transparent to traffic type and data protocol makes its integration into the existing access network far more rapid. Nonetheless, the atmospheric channel effects such as thick fog, smoke and turbulence as well as the attainment of $99.999 \%$ availability still pose the greatest challenges to long range terrestrial FSO. One practical solution is the deployment of a hybrid FSO/RF link, where an RF link acts as a backup to the FSO. 


\section{Fundamentals of FSO}

FSO in basic terms is the transfer of signals/data/information between two points using optical radiation as the carrier signal through an unguided channel. The data to be transported could be modulated on the intensity, phase or frequency of the optical carrier. An FSO link is essentially based on line-of sight (LOS). Thus, both the transmitter and the receiver must directly 'see' one another without any obstruction in their path for the communication link to be established. The unguided channels could be any or a combination of the space, sea-water, or the atmosphere. The emphasis here is on terrestrial FSO and as such only the atmospheric channel will be considered.

An FSO communication system can be implemented in two variants. The conventional FSO shown in Fig. 1 is for point-to-point communication with two similar transceivers; one at each end of the link. This allows for a full-duplex communication. The second variant uses the modulated retro-reflector (MRR). Laser communication links with MRRs are composed of two different terminals and hence are asymmetric links. On one end of the link, there is the MRR while the other hosts the interrogator as shown in Fig. 2. The interrogator projects a continuous wave $(\mathrm{CW})$ laser beam out to the retro-reflector. The modulated retro-reflector modulates the CW beam with the input data stream. The beam is then retro-reflected back to the interrogator. The interrogator receiver collects the return beam and recovers the data stream from it. The implementation just described permits only simplex communication. A two-way communication can also be achieved with the MRR by adding a photodetector to the MRR terminal and the interrogator beam shared in a half-duplex manner. Unless otherwise stated however, the conventional FSO link is assumed throughout this chapter.

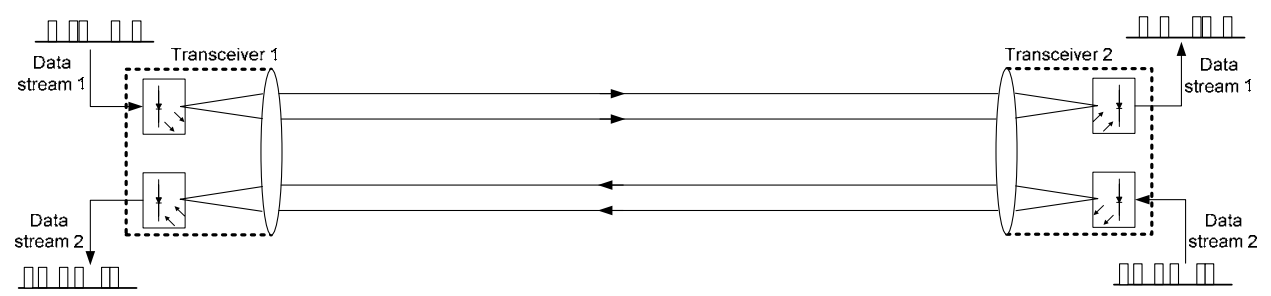

Fig. 1. Conventional FOS system block diagram

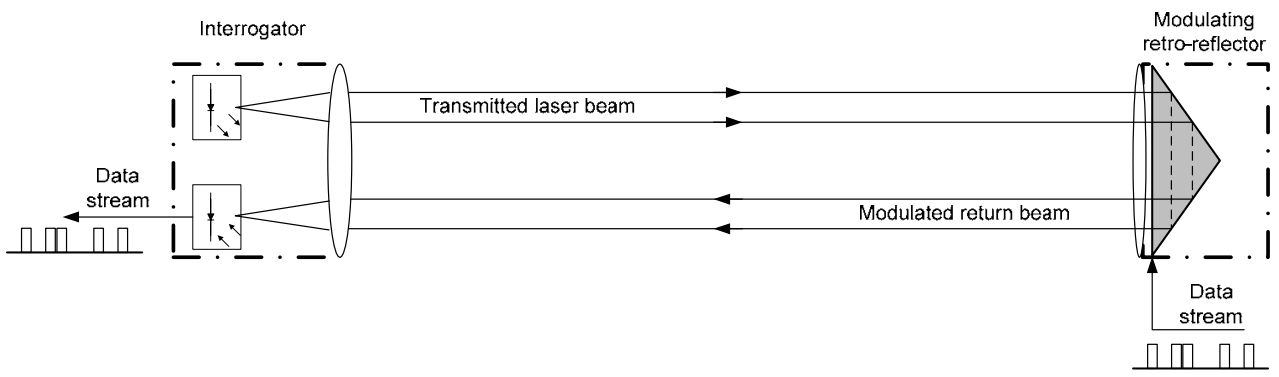

Fig. 2. Modulated retro-reflector based FSO system block diagram

The basic features of FSO, areas of application and the description of each fundamental block are further discussed in the following sections. 


\subsection{Features of FSO}

The basic features of the FSO technology are given below:

a) Huge modulation bandwidth - In general, the optical carrier frequency which includes infrared, visible and ultra violet frequencies are far greater than RF. And in any communication system, the amount of data transported is directly related to the bandwidth of the modulated carrier. The allowable data bandwidth can be up to $20 \%$ of the carrier frequency. Using optical carrier whose frequency ranges from $10^{12}-10^{16} \mathrm{~Hz}$ could hence permit up to $2000 \mathrm{THz}$ data bandwidth. Optical communication therefore, guarantees an increased information capacity. The usable frequency bandwidth in RF range is comparatively lower by a factor of $10^{5}$.

b) Narrow beam size - The optical radiation prides itself with an extremely narrow beam, a typical laser beam has a diffraction limit divergence of between $0.01-0.1$ mrad (Killinger, 2002). This implies that the transmitted power is only concentrated within a very narrow area. Thus providing FSO link with adequate spatial isolation from its potential interferers. The tight spatial confinement also allows for the laser beams to operate nearly independently, providing virtually unlimited degrees of frequency reuse in many environments and makes data interception by unintended users difficult. Conversely, the narrowness of the beam implies a tighter alignment requirement.

c) Unlicensed spectrum - Due to the congestion of the RF spectrum, interference from adjacent carriers is a major problem facing wireless RF communication. To minimise this interference, regulatory authorities put stringent regulations in place. To be allocated a slice of the RF spectrum therefore requires a huge fee and several months of bureaucracy. But the optical frequencies are free from all of this, at least for now. The initial set-up cost and the deployment time are then reduced and the return on investments begins to trickle in far more quickly.

d) Cheap - The cost of deploying FSO is lower than that of an RF with a comparable data rate. FSO can deliver the same bandwidth as optical fibre but without the extra cost of right of way and trenching. Based on a recent finding done by 'fSONA', an FSO company based in Canada, the cost per Mbps per month based on FSO is about half that of RF based systems (Rockwell and Mecherle, 2001).

e) Quick to deploy and redeploy - The time it takes for an FSO link to become fully operational starting from installation down to link alignment could be as low as four hours. The key requirement is the establishment of an unimpeded line of sight between the transmitter and the receiver. It can as well be taken down and redeployed to another location quite easily.

f) Weather dependent - The performance of terrestrial FSO is tied to the atmospheric conditions. The unfixed properties of the FSO channel undoubtedly pose the greatest challenge. Although this is not peculiar to FSO as RF and satellite communication links also experience link outages during heavy rainfall and in stormy weather. 
In addition to the above points, other secondary features of FSO include:

- It benefits from existing fibre optics communications optoelectronics

- It is free from and does not cause electromagnetic interference

- $\quad$ Unlike wired systems, FSO is a non-fixed recoverable asset

- $\quad$ The radiation must be within the stipulated safety limits

- $\quad$ Light weight and compactness

- Low power consumption

- Requires line of sight and strict alignment as a result of its beam narrowness.

\subsection{Areas of application}

The characteristic features of FSO discussed above make it very attractive for various applications within the access and the metro networks. It can conveniently complement other technologies (such as wired and wireless radio frequency communications, fibre-tothe- $X$ technologies and hybrid fibre coaxial among others) in making the huge bandwidth that resides in the optical fibre backbone available to the end users. Most end users are within a short distance from the backbone - one mile or less; this makes FSO very attractive as a data bridge between the backbone and the end-users. Among other emerging areas of application, terrestrial FSO has been found suitable for use in the following areas:

a) Last mile access - FSO can be used to bridge the bandwidth gap (last mile bottleneck) that exists between the end-users and the fibre optics backbone. Links ranging from $50 \mathrm{~m}$ up to a few $\mathrm{km}$ are readily available in the market with data rates covering $1 \mathrm{Mbps}$ to $2.5 \mathrm{Gbps}$ (Willebrand and Ghuman, 2002).

b) Optical fibre back up link - Used to provide back-up against loss of data or communication breakdown in the event of damage or unavailable of the main optical fibre link.

c) Cellular communication back-haul - Can be used to back-haul traffics between base stations and switching centres in the $3^{\text {rd }} / 4^{\text {th }}$ generation $(3 \mathrm{G} / 4 \mathrm{G})$ networks, as well as transporting IS-95 code division multiple access (CDMA) signals from macro-and microcell sites to the base stations.

d) Disaster recovery/Temporary links - The technology finds application where a temporary link is needed be it for a conference or ad-hoc connectivity in the event of a collapse of an existing communication network.

e) Multi-campus communication network - Can be used to interconnect campus networks

f) Difficult terrains - For example across a river, very busy street, rail tracks or where right of way is not available or too expensive to pursue, FSO is an attractive data bridge in such instances.

\section{FSO Block Diagram}

The block diagram of a typical terrestrial FSO link is shown in Fig. 3. Like any other communication technologies, the FSO essentially comprises of three parts: the transmitter, 
the channel and the receiver. These basic parts are further discussed in the sections that follow.

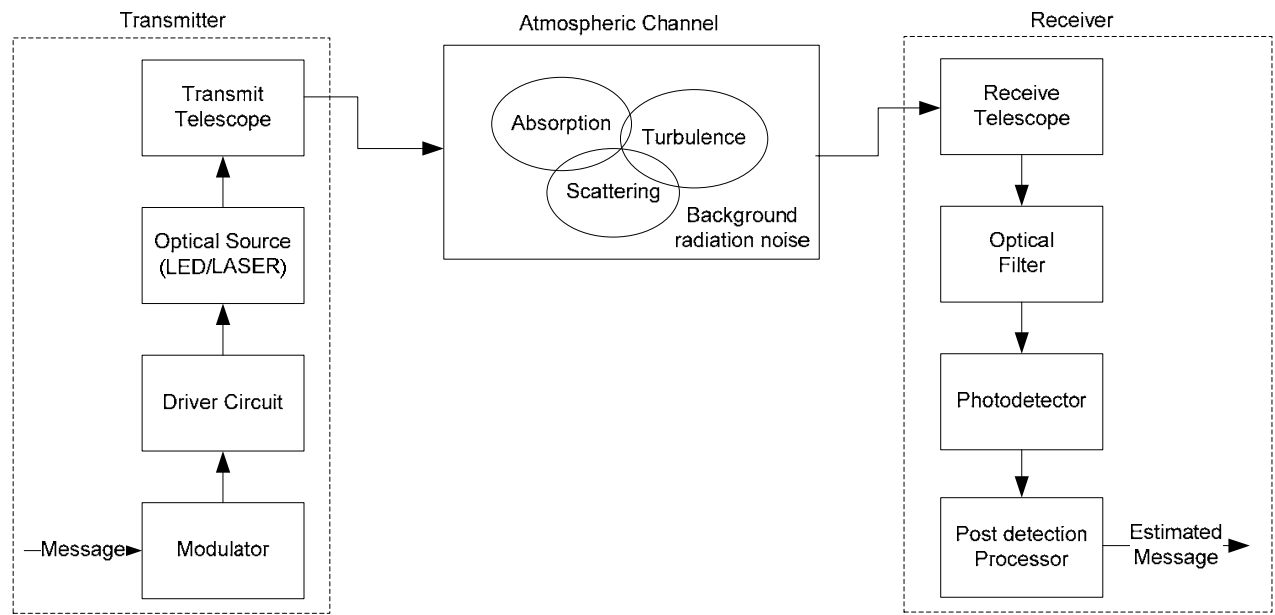

Fig. 3. Block diagram of a terrestrial FSO link

\subsection{The transmitter}

This functional element has the primary duty of modulating the source data onto the optical carrier which is then propagated through the atmosphere to the receiver. The most widely used modulation type is the intensity modulation (IM) in which the source data is modulated on the irradiance/intensity of the optical radiation. This is achieved by varying the driving current of the optical source directly in sympathy with the data to be transmitted or via an external modulator such as the symmetric Mach-Zehnder (SMZ) interferometer. The use of an external modulator guarantees a higher data rate than what is obtainable with direct modulation but an external modulator has a nonlinear response. Other properties of the radiated optical field such as its phase, frequency and state of polarisation can also be modulated with data/information through the use of an external modulator. The transmitter telescope collects, collimates and directs the optical radiation towards the receiver telescope at the other end of the channel. Table 1 presents a summary of commonly used sources in FSO systems. 


\begin{tabular}{|c|c|c|}
\hline Wavelength (nm) & Type & Remark \\
\hline$\sim 850$ & $\begin{array}{l}\text { Vertical cavity surface } \\
\text { emitting laser }\end{array}$ & $\begin{array}{l}\text { Cheap and readily available (CD lasers) } \\
\text { No active cooling } \\
\text { Lower power density } \\
\text { Reliable up to } \sim 10 \mathrm{Gbps}\end{array}$ \\
\hline$\sim 1300 / \sim 1550$ & $\begin{array}{c}\text { Fabry-Perot } \\
\text { Distributed-feedback } \\
\text { lasers }\end{array}$ & $\begin{array}{l}\text { Long life } \\
\text { Lower eye safety criteria } \\
50 \text { times higher power density }(100 \\
\left.\mathrm{mW} / \mathrm{cm}^{2}\right) \\
\text { Compatible with EDFA } \\
\text { High speed, up to } 40 \mathrm{Gbps} \\
\text { A slope efficiency of } 0.03-0.2 \mathrm{~W} / \mathrm{A}\end{array}$ \\
\hline$\sim 10,000$ & Quantum cascade laser & $\begin{array}{l}\text { Expensive and relative new } \\
\text { Very fast and highly sensitive } \\
\text { Better fog transmission characteristics. } \\
\text { Components not readily available } \\
\text { No penetration through glass }\end{array}$ \\
\hline Near Infrared & LED & $\begin{array}{l}\text { Cheaper } \\
\text { Simpler driver circuit } \\
\text { Lower power and lower data rates }\end{array}$ \\
\hline
\end{tabular}

Table 1. Optical sources

Within the 700-10,000 nm wavelength band there are a number transmission windows that are almost transparent with an attenuation of $<0.2 \mathrm{~dB} / \mathrm{km}$. The majority of FSO systems are designed to operate in the 780-850 $\mathrm{nm}$ and $1520-1600 \mathrm{~nm}$ spectral windows. $780 \mathrm{~nm}-850$ $\mathrm{nm}$ is the most widely used because devices and components are readily available in this wavelength range and at low cost. The $1550 \mathrm{~nm}$ band is attractive for a number of reasons i) compatibility with the $3^{\text {rd }}$ window wavelength-division multiplexing networks, ii) eye safety (about 50 times more power can be transmitted at $1550 \mathrm{~nm}$ than at $850 \mathrm{~nm}$ ), and iii) reduced solar background and scattering in light haze/fog. Consequently, at $1550 \mathrm{~nm}$ a significantly more power can be transmitted to overcome attenuation by fog. However, the drawbacks of the $1550 \mathrm{~nm}$ band are slightly reduced detector sensitivity, higher component cost and a stricter alignment requirement.

\subsection{The receiver}

The receiver helps recover the transmitted data from the incident optical field. The receiver is composed of:

a) The receiver telescope - collects and focuses the incoming optical radiation on to the photodetector. It is should be noted that a large receiver telescope aperture is desirable as it collects multiple uncorrelated radiations and focuses their average on the photodetector. This is referred to as aperture averaging but a wide aperture also means more background radiation/noise,

b) An optical band - pass filter to reduce the amount of background radiations,

c) A photodetector - PIN or APD that converts the incident optical field into an electrical signal. The commonly used photodetector for in the contemporary laser 
communication systems are summarised in Table 2. Germanium only detectors are generally not used in FSO because of their high dark current.

d) Post-detection processor/decision circuit - where the necessary amplification, filtering and signal processing necessary to guarantee a high fidelity data recovery are carried out.

Due to detector capacitance effect, higher speed detectors are inherently smaller in size (70 $\mu \mathrm{m}$ and $30 \mu \mathrm{m}$ for $2.5 \mathrm{Gbps}$ and $10 \mathrm{Gbps}$, respectively) with a limited field-of-view (FOV) that require accurate alignment. FOV of the receiver is the ratio of the detector size to the focal length (Jeganathan and Ionov): $F O V=d / f=d F \# / D$; where $d$ is the detector diameter, $f$ is the effective focal length, and $D$ is the receiver aperture. The quantity $F \#$ is the f-number. For a $75 \mu \mathrm{m}$ size detector, with $F \#=1$ and $D=150 \mathrm{~mm}$ telescope, the FOV $=\sim 0.5 \mathrm{mrad}$.

\begin{tabular}{|l|l|l|l|l|}
\hline Material/Structure & $\begin{array}{c}\text { Wavelength } \\
\text { (nm) }\end{array}$ & Responsivity & Typical Sensitivity & Gain \\
\hline Silicon PIN & $300-1100$ & 0.5 & $-34 \mathrm{dBm} @ 155 \mathrm{Mbps}$ & 1 \\
Silicon PIN, with & $300-1100$ & 0.5 & $-26 \mathrm{dBm} @ 1.25 \mathrm{Gbps}$ & 1 \\
$\begin{array}{l}\text { Transimpedance } \\
\text { amplifier }\end{array}$ & & & & \\
InGaAs PIN & $1000-1700$ & 0.9 & $-46 \mathrm{dBm} @ 155 \mathrm{Mbps}$ & 1 \\
Silicon APD & $400-1000$ & 77 & $-52 \mathrm{dBm} @ 155 \mathrm{Mbps}$ & 150 \\
InGaAs APD & $1000-1700$ & 9 & $-33 \mathrm{dBm} @ 1.25 \mathrm{Gbps}$ & 10 \\
$\begin{array}{l}\text { Quantum-well and } \\
\text { Quatum-dot detectors }\end{array}$ & 10,000 & & & \\
\hline
\end{tabular}

Table 2. FSO Photodetectors

The receiver detection process can be classified into:

a) Direct detection receiver - This type of receiver detects the instantaneous intensity or power of the optical radiation impinging on the photodetector. Hence, the output of the photodetector is proportional to the power of the incident field. Its implementation is very simple and most suitable for intensity modulated optical systems (Gagliardi and Karp, 1995, Pratt, 1969). The block diagram of direct detection receiver is shown in Fig. 4.

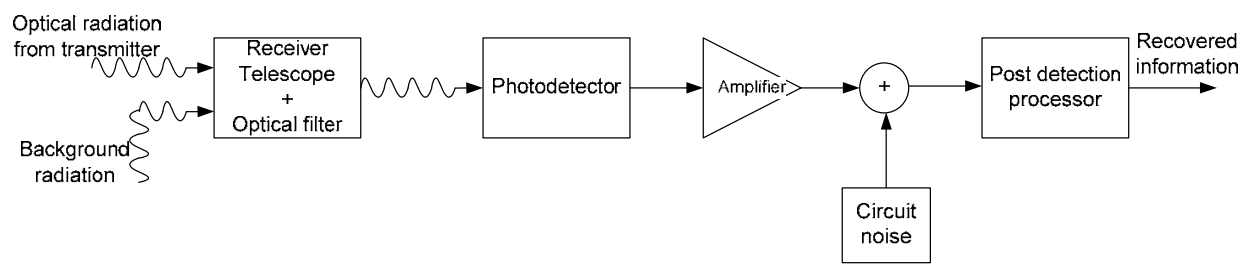

Fig. 4. The block diagram of a direct detection optical receiver. 
b) Coherent detection receiver - The coherent receiver whose block diagram is shown in Fig. 5 works based on the photo-mixing phenomenon. The incoming optical field is mixed with another locally generated optical field on the surface of the photodetector. The coherent receiver can be further divided into homodyne and heterodyne receivers. In homodyne receivers, the frequency/wavelength of the local (optical) oscillator is exactly the same as that of the incoming radiation while in heterodyne detection, the incoming radiation and the local oscillator frequencies are different. In contrast to the RF coherent detection, the output of the local oscillator in an optical coherent detection is not required to have the same phase as the incoming radiation. The principal advantages of a coherent receiver are: relative ease of amplification at an intermediate frequency and the fact that the signal-to-noise ratio can be significantly improved by simply raising the local oscillator power.

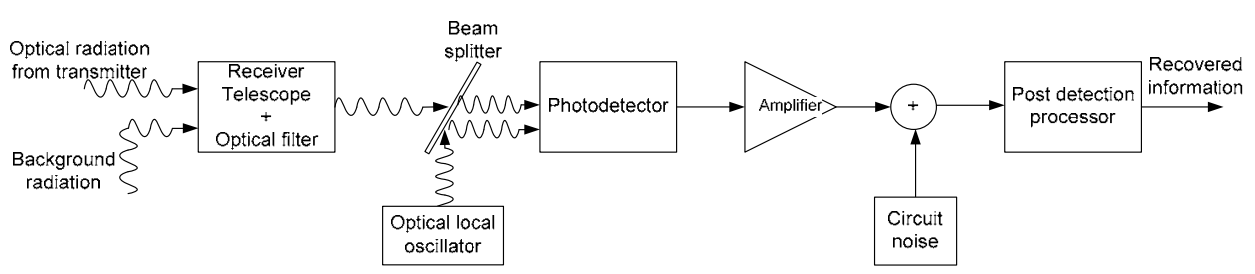

Fig. 5. The block diagram of a coherent detection optical receiver.

\subsection{The atmospheric channel}

An optical communications channel differs from the conventional Gaussian-noise channel, in that the channel input signal $x(t)$ represents power rather than amplitude. This leads to two constraints on the transmitted signal: i) $x(t)$ must be non-negative, and ii) the average value of $x(t)$ must not exceed a specified value $P_{\max } \geq \lim _{T \rightarrow \infty} \frac{1}{2 T} \int_{-T}^{T} x(t) d t$. In contrast to the conventional channels, where the signal-to-noise ratio (SNR) is proportional to the power, in optical systems the received electrical power and the variance of the shot noise are proportional to $A_{d}^{2}$ and $A_{d}$, respectively; where $A_{d}$ is the receiver detector area. Thus, for a shot noise limited optical system, the SNR is proportional to $A_{d}$. This implies that for a given transmit power; a higher SNR can be attained by using a large area detector. However, as $A_{d}$ increases so does its capacitance, which has a limiting effect on the receiver bandwidth. The atmospheric channel consists of gases (see Table 3), and aerosols - tiny particles suspended in the atmosphere. Also present in the atmosphere are rain, haze, fog and other forms of precipitation. The amount of precipitation present in the atmosphere depends on the location (longitude and latitude) and the season. The highest concentration of particles is obviously near the Earth surface within the troposphere; this decreases with increasing altitude up through to the ionosphere (Gagliardi and Karp, 1995). 


\begin{tabular}{|lll|}
\hline Constituent & Volume Ratio (\%) & Parts Per Million (ppm) \\
\hline Nitrogen $\left(\mathrm{N}_{2}\right)$ & 78.09 & \\
Oxygen $\left(\mathrm{O}_{2}\right)$ & 20.95 & \\
Argon $(\mathrm{Ar})$ & 0.93 & \\
Carbon dioxide $\left(\mathrm{CO}_{2}\right)$ & 0.03 & $40-40,000$ \\
Water vapour $\left(\mathrm{H}_{2} \mathrm{O}\right)$ & & 20 \\
Neon $(\mathrm{Ne})$ & & 5.2 \\
Helium $(\mathrm{He})$ & & 1.5 \\
Methane $\left(\mathrm{CH}_{4}\right)$ & 1.1 \\
Krypton $(\mathrm{Kr})$ & 1 \\
Hydrogen $\left(\mathrm{H}_{2}\right)$ & 0.6 \\
Nitrous oxide $\left(\mathrm{N}_{2} \mathrm{O}\right)$ & 0.2 \\
Carbon monoxide $(\mathrm{CO})$ & & 0.05 \\
Ozone $\left(\mathrm{O}_{3}\right)$ & & 0.09 \\
Xenon $($ Xe) & & \\
\hline
\end{tabular}

Table 3. The gas constituents of the atmosphere (AFGL, 1986).

Another feature of interest is the atmospheric turbulence. When radiation strikes the Earth from the Sun, some of the radiation is absorbed by the Earth's surface thereby heating up its (Earth's) surface air mass. The resulting mass of warm and lighter air then rises up to mix turbulently with the surrounding cooler air mass to create atmospheric turbulence. This culminates in small (in the range of 0.01 to 0.1 degrees) but spatially and temporally fluctuating atmospheric temperature (Killinger, 2002). With the size distribution of the atmospheric constituents ranging from sub-micrometres to centimetres, an optical field that traverses the atmosphere is scattered and or absorbed resulting in the following:

\subsubsection{Power loss}

For an optical radiation traversing the atmosphere, some of the photons are extinguished (absorbed) by the molecular constituents (water vapour, $\mathrm{CO}_{2}$, fog, ozone etc) and their energy converted into heat while others experience no loss of energy but their initial direction of propagation are changed (scattering). The Beer-Lambert law describes the transmittance of an optical field through the atmosphere. The beam also spreads out while traversing the channel causing the size of the received beam to be greater than the receiver aperture. These factors, combined with others herein discussed are responsible for the difference between the transmitted and the received optical powers.

\subsubsection{Atmospheric channel loss}

The atmospheric channel attenuates the field traversing it as a result of absorption and scattering processes. The concentrations of matter in the atmosphere, which result in the signal attenuation vary spatially and temporally, and will depend on the current local weather conditions. For a terrestrial FSO link transmitting optical signal through the atmosphere, the received irradiance at a distance, $L$ from the transmitter is related to the transmitted irradiance by the Beer-Lambert's law given as (Gagliardi and Karp, 1995): 


$$
\tau(\lambda, L)=\frac{P_{R}}{P_{T}}=\exp [-\gamma(\lambda) L]
$$

where $\gamma(\lambda)$ and $\tau(\lambda, L)$ represent the total attenuation/extinction coefficient $\left(\mathrm{m}^{-1}\right)$ and the transmittance of the atmosphere at wavelength $\lambda$, respectively. The attenuation of the optical signal in the atmosphere is due to the presence of molecular constituents (gases) and aerosol. The attenuation coefficient is the sum of the absorption and the scattering coefficients from aerosols and molecular constituents of the atmosphere, it follows therefore that (Willebrand and Ghuman, 2002):

$$
\gamma(\lambda)=\alpha_{m}(\lambda)+\alpha_{a}(\lambda)+\beta_{m}(\lambda)+\beta_{a}(\lambda)
$$

The first two terms represent the molecular and aerosol absorption coefficients, respectively while the last two terms are the molecular and aerosol scattering coefficients respectively.

a) Absorption - This takes place when there is an interaction between the propagating photons and molecules (present in the atmosphere) along its path. Some of the photons are extinguished and their energies converted into heat (Pratt, 1969). The absorption coefficient depends very much on the type of gas molecules and their concentration (Gagliardi and Karp, 1995). Absorption is wavelength dependent and therefore selective. This leads to the atmosphere having transparent zones range of wavelengths with minimal absorptions - referred to as the transmission windows. However, the wavelengths used in FSO are basically chosen to coincide with the atmospheric transmission windows (Bloom et al., 2003), resulting in the attenuation coefficient being dominated by scattering. Hence, $\gamma(\lambda) \cong \beta_{a}(\lambda)$.

b) Scattering - Results in angular redistribution of the optical field with and without wavelength modification. The scattering effect depends on the radius, $r$ of the particles (fog, aerosol) encountered during propagation. One way of describing this is to consider the size parameter $x_{o}=2 \pi r / \lambda$. If $x_{o} \ll 1$, the scattering process is classified as Rayleigh scattering (Bates, 1984); if $x_{o} \approx 1$ it is Mie scattering and for $x_{o} \gg 1$, the scattering process can then be explained using the diffraction theory (geometric optics). The scattering process for different scattering particles present in the atmosphere is summarised in Table 4.

\begin{tabular}{|l|l|l|l|}
\hline Type & Radius $(\mu \mathrm{m})$ & Size Parameter $x_{o}$ & Scattering Process \\
\hline Air Molecules & 0.0001 & 0.00074 & Rayleigh \\
Haze particle & $0.01-1$ & $0.074-7.4$ & Rayleigh - Mie \\
Fog droplet & $1-20$ & $7.4-147.8$ & Mie - Geometrical \\
Rain & $100-10000$ & $740-74000$ & Geometrical \\
Snow & $1000-5000$ & $7400-37000$ & Geometrical \\
Hail & $5000-50000$ & $37000-370000$ & Geometrical \\
\hline
\end{tabular}

Table 4. Typical atmospheric scattering particles with their radii and scattering process at $\lambda$ $=850 \mathrm{~nm}$ 
The fog particle size compares very much with the wavelength band of interest in FSO 0.5 $\mu \mathrm{m}-2 \mu \mathrm{m})$. Thereby making fog a major photon scatterer and it contributes the most optical power attenuation. The Mie scattering will be described based on empirical formulae expressed in terms of the visibility range $V$ in $\mathrm{km}$. The visibility range is the distance that a parallel luminous beam travels through in the atmosphere until its intensity drops to $2 \%$ of its original value (Willebrand and Ghuman, 2002). The visibility is measured with an instrument called the transmissiometer. A common empirical model for Mie scattering is given by:

$$
\beta_{a}(\lambda)=\frac{3.91}{V}\left(\frac{\lambda}{550}\right)^{-\delta}
$$

where $\delta$ is given as:

$$
\delta=\left\{\begin{array}{lc}
1.6 & V>50 \\
1.3 & 6<V<50 \\
0.16 V+0.34 & 1<V<6 \\
V-0.5 & 0.5<V<1 \\
0 & V<0.5
\end{array} \quad \delta=\left\{\begin{array}{lr}
1.6 & V>50 \\
1.3 & 6<V<50 \\
0.585 V^{1 / 3} & V<6
\end{array}\right.\right.
$$

Given in Table 5 are the visibility range values under different weather conditions.

\begin{tabular}{|l|c|}
\hline Weather Condition & Visibility Range $(\mathbf{m})$ \\
\hline Thick fog & 200 \\
Moderate fog & 500 \\
Light fog & $770-1000$ \\
Thin fog/heavy rain $(25 \mathrm{~mm} / \mathrm{hr})$ & $1900-2000$ \\
Haze/medium rain $(12.5 \mathrm{~mm} / \mathrm{hr})$ & $2800-40000$ \\
Clear/drizzle $(0.25 \mathrm{~mm} / \mathrm{hr})$ & $18000-20000$ \\
Very clear & $23000-50000$ \\
\hline
\end{tabular}

Table 5. Weather conditions and their visibility range values

Recently, Al Naboulsi (al Naboulsi and Sizun, 2004) in his work came up with a simple relationship for advection and radiation fog attenuation in the $690-1550 \mathrm{~nm}$ wavelength range in the visibility range $50-1000 \mathrm{~m}$ as:

$$
\begin{gathered}
\alpha_{\text {Advection }}(\lambda)=\frac{0.11478 \lambda+3.8367}{V} \\
\alpha_{\text {Radiation }}(\lambda)=\frac{0.18126 \lambda^{2}+0.13709 \lambda+3.7502}{V}
\end{gathered}
$$


where $\lambda$ is the wavelength in $\mathrm{nm}$ and the visibility $V$ is in metres. The power loss due to rain and snow are so low compared to that due to the Mie scattering. But they still have to be accounted for in the link margin during the link budget analysis. A typical rainfall of 2.5 $\mathrm{cm} /$ hour could result in an attenuation of $\sim 6 \mathrm{~dB} / \mathrm{km}$ (Kim and Korevaar, 2001) while a typical value for attenuation due to light snow to blizzard is $3 \mathrm{~dB} / \mathrm{km}$ to $30 \mathrm{~dB} / \mathrm{km}$ (Willebrand and Ghuman, 2002). In early 2008 in Prague, Czech Republic, the fog attenuation was measured and compared with the empirical fog attenuation models. This result is shown in Fig. 6; with a visibility of less than $200 \mathrm{~m}$ - thick fog - the recorded fog attenuation is $\sim 200 \mathrm{~dB} / \mathrm{km}$. All the empirical models provide a reasonable fit to the measured values with a maximum of about $\pm 5 \mathrm{~dB} / \mathrm{km}$ difference between any two empirical models.

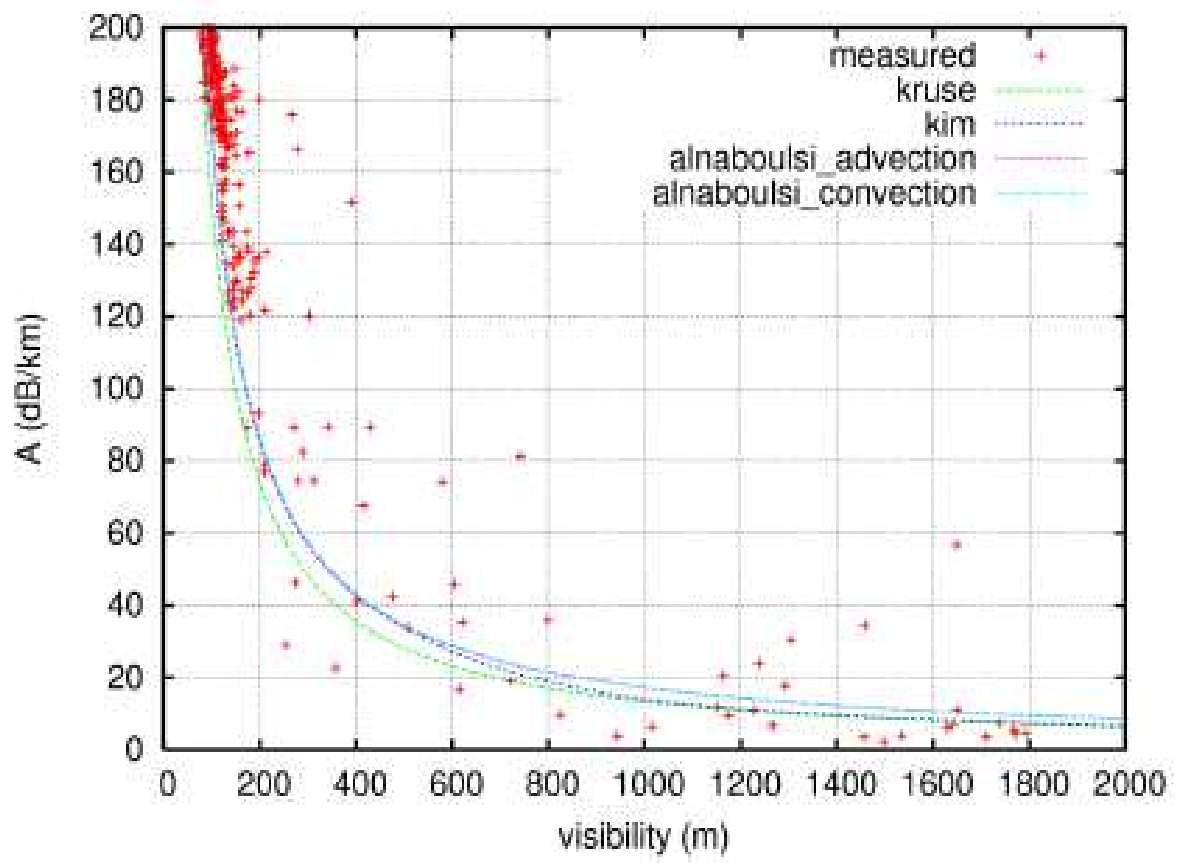

Fig. 6. Attenuation coefficient as a function of visibility range at $\lambda=830 \mathrm{~nm}$ (Grabner and Kvicera, 2009).

\subsubsection{Beam divergence}

One of the main advantages of FSO systems is the ability to transmit a very narrow optical beam, thus offering enhanced security. But due to diffraction, the beam spreads out. This results in a situation in which the receiver aperture is only able to collect a fraction of the beam and hence beam divergence loss. 


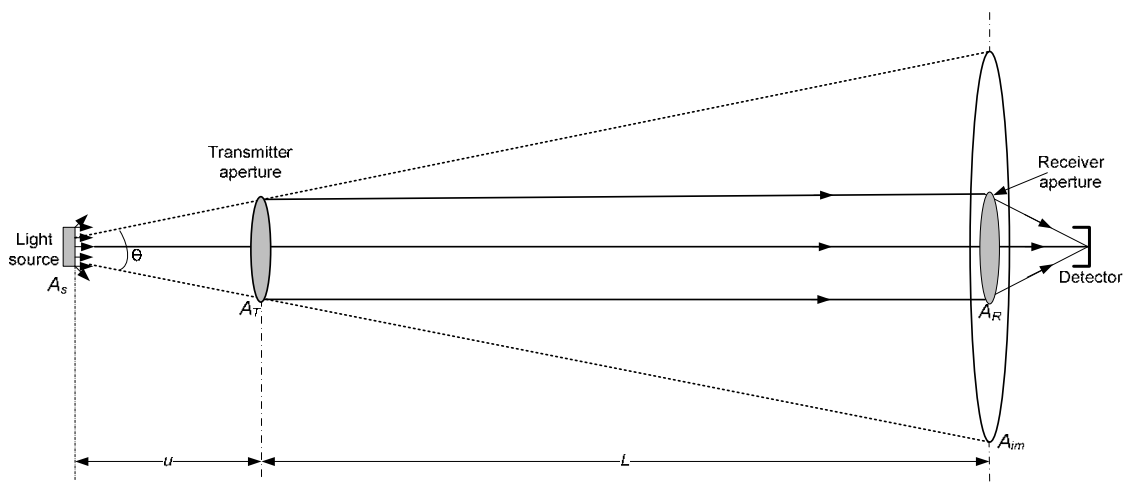

Fig. 7. Beam divergence

Considering the arrangement of a free-space optical communication link of Fig. 7, and by invoking the thin lens approximation to the diffuse optical source whose irradiance is represented by $I_{s}$, the amount of optical power focused on the detector is derived as (Gowar, 1993):

$$
P_{R}=\frac{I_{S} A_{T} A_{R}}{L^{2} A_{S}}
$$

$A_{T}$ and $A_{R}$ are the transmitter and receiver aperture areas while $A_{s}$ is the area of the optical source. This clearly shows that a source with high radiance $I_{s} / A_{s}$ and wide apertures are required in order to increase the received optical power.

For a non-diffuse, small source such as the laser, the size of the image formed at the receiver plane is no longer given by the thin lens approximation; it is determined by diffraction at the transmitter aperture. The diffraction pattern produced by a uniformly illuminated circular aperture of diameter, $d_{T}$ is known to consists of a set of concentric rings. The image size is said to be diffraction limited when the radius of the first intensity minimum or dark ring of the diffraction pattern becomes comparable in size with the diameter, $d_{i m}$ of the normally focussed image (Gowar, 1993). That is:

$$
d_{i m}=\frac{L}{u} d_{s}<1.22 \frac{\lambda L}{d_{T}}
$$

Therefore,

$$
d_{s}<1.22 \frac{\lambda u}{d_{T}} \approx 1.22 \frac{\lambda f}{d_{T}}
$$

This equation shows that for diffraction to be the sole cause of beam divergence (diffraction limited), the source diameter, $d_{s}<1.22 \frac{\lambda f}{d_{T}}$. Laser being inherently collimated and coherent normally produces a diffraction-limited image. The diffraction limited beam divergence angle in radian is given by $\theta_{b} \cong \frac{\lambda}{d_{T}}$. 
If the transmitter and receiver effective antenna gains are respectively given by:

$$
\begin{gathered}
G_{T}=\frac{4 \pi}{\Omega_{\mathrm{b}}} \\
G_{R}=\frac{4 \pi A_{T}}{\lambda^{2}}
\end{gathered}
$$

And the free-space path loss is given by:

Hence the received optical power is:

$$
\mathcal{L}=\left(\frac{\lambda}{4 \pi L}\right)^{2}
$$

$$
\begin{gathered}
P_{R}=P_{T} \mathcal{L} G_{T} G_{R} \\
P_{R}=P_{T} \frac{4 A_{R}}{L^{2} \Omega_{b}} \\
P_{R} \cong P_{T}\left(\frac{4}{\pi}\right)^{2} \frac{A_{T} A_{R}}{L^{2} \lambda^{2}}
\end{gathered}
$$

where the radiation solid angel $\Omega_{b} \cong \frac{\pi \theta_{b}^{2}}{4}$. The diffraction limited beam spreading /geometric loss in $\mathrm{dB}$ is thus:

$$
L_{\text {Geom }}=-10\left[\log \left(\frac{A_{T} A_{R}}{L^{2} \lambda^{2}}\right)+2 \log \left(\frac{4}{\pi}\right)\right]
$$

The result given by (13) can be obtained by substituting $A_{i m}=\theta_{b} L$ for the image size in $P_{R}=P_{T} \frac{A_{R}}{A_{i m}}$. A beam expander of the type shown in Fig. 8 , in which the diffracting aperture has been increased, could then be used to reduce the diffraction-limited beam divergence. Thereby reducing the beam divergence loss and increasing the received power in the process.

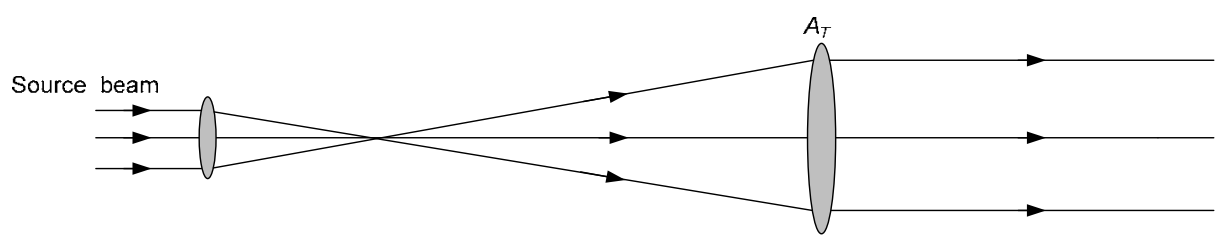

Fig. 8. Beam expander diagram

However for most practical sources, the beam divergence angle is usually greater than that dictated by diffraction. For a source with an angle of divergence $\theta$, the beam size at a 
distance $L$ away is $\left(d_{T}+\theta L\right)$. The fraction of the received power to the transmitted power is therefore be given as:

$$
\frac{P_{R}}{P_{T}}=\frac{A_{R}}{A_{I m}}=\frac{d_{R}^{2}}{\left(d_{T}+\theta L\right)^{2}}
$$

And the geometric loss in $\mathrm{dB}$ thus becomes:

$$
L_{\text {Geom }}=-20 \log \left[\frac{d_{R}}{\left(d_{T}+\theta L\right)}\right]
$$

The beam spreading loss for the diffraction limited source given by (13) is expectedly lower than for the non-diffraction limited case given by (15), since the image size is smaller by $d_{T}$ in the diffraction limited case.

From the foregoing, a source with very narrow divergence beam angle is preferable. It should however be mentioned that wide divergence angles are desirable in short range FSO links to ease the alignment requirement, compensate for building sway and eliminate the need for active tracking systems at the expense of increased geometric loss apparently. A typical FSO transceiver has optical beam divergence in the range of 2-10 mrad and 0.05-1.0 mrad (equivalent to a beam spread of $2-10 \mathrm{~m}$, and $5 \mathrm{~cm}$ to $1 \mathrm{~m}$, respectively at $1 \mathrm{~km}$ link range) for systems without and with tracking, respectively.

\subsubsection{Optical and window loss}

This type of loss includes losses due to imperfect lenses and other optical elements used in the design of both the transmitter and receiver. It accounts for the reflection, absorption and scattering due to the lenses in the system (Willebrand and Ghuman, 2002). The value of the optical loss $L_{O}$ can be obtained from the component manufacturer. It apparently depends on the characteristics of the equipments and the quality of the lenses used. For FSO transceivers installed behind windows within a building, there exists an additional optical power loss due the window glass attenuation. Although (glass) windows allow optical signals to pass through them, they contribute to the overall power loss of the signal. Uncoated glass windows usually attenuate $4 \%$ per surface, because of reflection. Coated windows display much higher losses and its magnitude is wavelength dependent.

\subsubsection{Pointing loss}

Additional power penalty is usually incurred due to lack of perfect alignment of the transmitter and receiver. The resulting power loss is catered for by including pointing/misalignment loss, $L_{P}$ in the link budget analysis. For short FSO links $(<1 \mathrm{~km})$, this might not be an issue but for longer link ranges, this can certainly not be neglected. Misalignments could result from building sway or strong wind effect on the FOS link head stands.

\subsubsection{The link budget}

Based on the losses mentioned above, the received optical power in $\mathrm{dBm}$ can thus be obtained from the link budget equation as: 


$$
P_{R}(\lambda, L)=P_{T}(\lambda, 0)-4.343 L \beta_{a}(\lambda)-L_{G e o m}-L_{O}-L_{P}-L_{M}
$$

The link margin, $L_{M}$ is included in the link budget equation above to cater for other losses such as changes in specification when a faulty component is replaced, ageing of laser sources, attenuation due to rain, snow and so on. Figure 9 depicts the link range against available link margin at different values of visibility for a typical commercial FSO link whose parameters are tabulated in Table 6. In this figure, the Kim model is used in estimating the attenuation coefficient. By operating the link under consideration at a $5 \mathrm{~dB}$ link margin in clear atmosphere with over $30 \mathrm{~km}$ visibility, two data nodes at about $3 \mathrm{~km}$ apart and running at $155 \mathrm{Mbps}$ can be reliably connected with an FSO system whose parameters are shown in Table 6.

\begin{tabular}{|l|l|}
\hline Parameter & Typical Value \\
\hline Receiver aperture diameter $\left(\mathrm{d}_{\mathrm{R}}\right)$ & $8 \mathrm{~cm}$ \\
Transmitter aperture diameter $\left(\mathrm{d}_{\mathrm{T}}\right)$ & $2.5 \mathrm{~cm}$ \\
Beam divergence $(\theta)$ & $2 \mathrm{mrad}$ \\
Modulation technique/Bit rate & On-OFF \\
& keying/155Mbps \\
Transmit power & $14 \mathrm{dBm}$ \\
Receiver sensitivity & $-30 \mathrm{dBm}$ \\
Optical loss $\left(L_{O}\right)$ & $1 \mathrm{~dB}$ \\
Pointing loss $\left(L_{P}\right)$ & $1 \mathrm{~dB}$ \\
Wavelength $(\lambda)$ & $850 \mathrm{~nm}$ \\
\hline
\end{tabular}

Table 6. Typical link budget parameters

One major importance of the link budget equation is in determining the achievable link range, for a given receiver sensitivity. The receiver sensitivity by the way represents the minimum amount of optical power needed for the system to achieve a specified level performance; for example a bit error rate of $10^{-9}$. The receiver sensitivity depends on the modulation technique in use, the noise level, fading/scintillation strength and the data rate. Higher data rate simply implies shorter optical pulse duration, hence fewer photons that can be detected. The noise could be from a combination of background radiation, the detection process/quantum shot noise and the thermal noise caused by the thermal agitation of electrons in the receiver electronic components. The theoretical receiver sensitivity at any desired level of performance can be obtained from the analysis of Section 5 . 


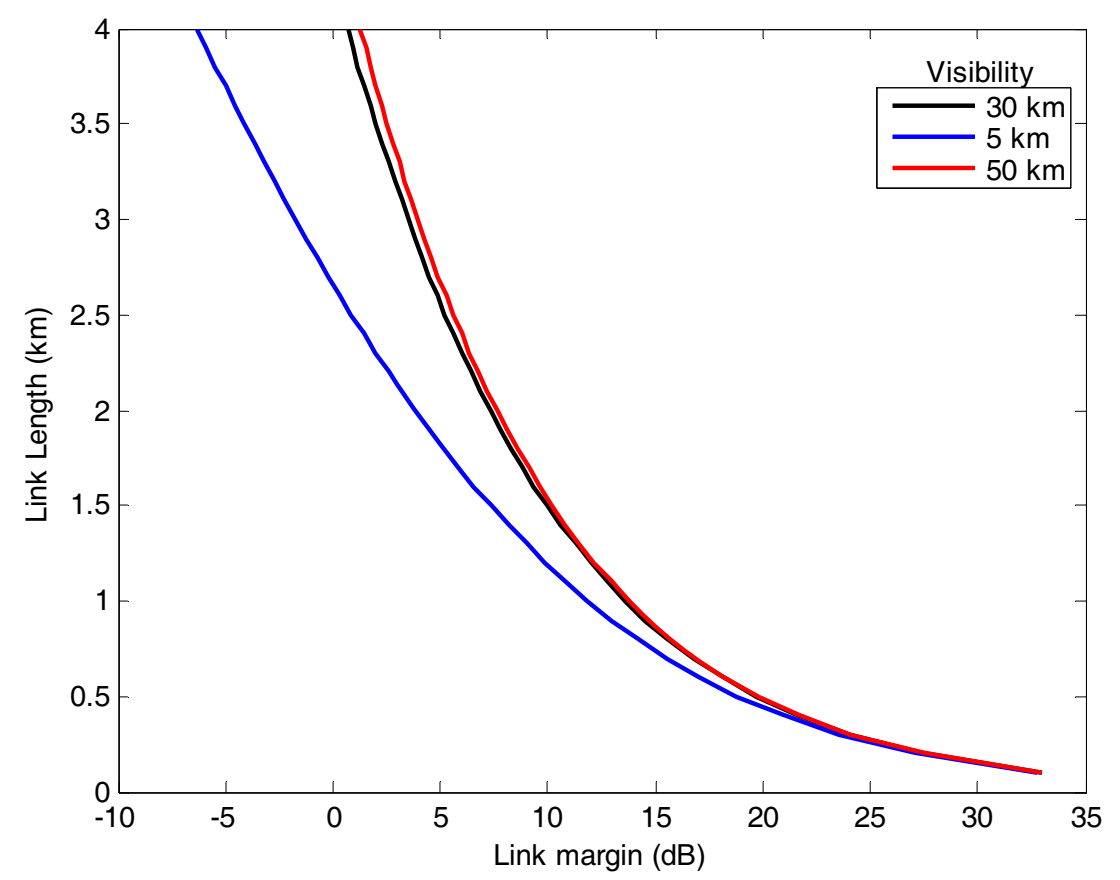

Fig. 9. Link length against available link margin for different visibility values.

\subsubsection{The atmospheric turbulence effects}

The temperature inhomogeneity of the atmosphere causes corresponding changes in the index of refraction of the atmosphere resulting in eddies, cells or air packets having varying sizes from $\sim 0.1 \mathrm{~cm}$ to $\sim 10 \mathrm{~m}$. These air packets act like refractive prisms of varying indices of refraction. The propagating optical radiation is therefore fully or partially deviated depending on the relative size of the beam and the degree of temperature inhomogeneity along its path. Consequently, the optical radiation traversing the turbulence atmosphere experiences random variation/fading in its irradiance (scintillation) and phase. Familiar effects of turbulence include the twinkling of stars caused by random fluctuations of stars' irradiance and the shimmer of the horizon on a hot day caused by random changes in the optical phase of the light beam resulting in the reduced image resolution (Killinger, 2002). Atmospheric turbulence depends on i) atmospheric pressure/altitude, ii) wind speed, and iii) variation of index of refraction due to temperature inhomogeneity. Known effects of atmospheric turbulence include (Pratt, 1969):

a) Beam steering - Angular deviation of the beam from its original LOS causing the beam to miss the receiver.

b) Image dancing - The received beam focus moves in the image plane due to variations in the beam's angle of arrival.

c) Beam spreading - Increased beam divergence due to scattering. This leads to a reduction in received power density. 
d) Beam scintillation - Variations in the spatial power density at the receiver plane caused by small scale destructive interference within the optical beam.

e) Spatial coherence degradation - Turbulence also induces losses in phase coherence across the beam phase fronts. This is particularly deleterious for photomixing (e.g. in coherent receiver).

f) Polarisation fluctuation - This results from changes in the state of polarisation of the received optical field after passing through a turbulent medium. However, the amount of polarisation fluctuation is negligible for a horizontally travelling optical radiation in atmospheric turbulence (Karp et al., 1988).

\subsubsection{Atmospheric turbulence model}

Atmospheric turbulence results from random fluctuation of the atmospheric refractive index $n$ along the path of a wave traversing the atmosphere. This refractive index fluctuation is the direct product of random variations in atmospheric temperature along the wave path. The random temperature changes themselves are a function of the altitude, $h$ and the wind speed, $v$. Scintillation causes impairment and performance degradation for long range (> $1 \mathrm{~km})$ atmospheric optical communication systems. The relationship between the temperature of the atmosphere and its refractive index is given by (Karp et al., 1988):

$$
n=1+77.6\left(1+7.52 \times 10^{-3} \lambda^{-2}\right) \frac{P}{T_{e}} \times 10^{-6}
$$

where $P$ is the atmospheric pressure in millibars, and $T_{e}$ is the temperature in Kelvin.

The turbulence atmosphere can be described as containing loosely packed eddies/prisms of varying sizes and refractive indices. The smallest eddy size $l_{0}$ is called the turbulence inner scale, with a value of a few millimetres, while the outer scale of turbulence $L_{o}$ has its value running to several meters. According to the Taylor's 'frozen-in' model, the temporal variation in statistical properties of the turbulent atmosphere is caused by the airmass movement. Also, the turbulent eddies are fixed and only vary with the wind moving perpendicularly to the direction of the traversing wave. The temporal coherence time $\tau_{\mathrm{o}}$ of atmospheric turbulence is known to be in the order of millisecond. This value is very large compared to typical data symbol duration. Hence the terrestrial FSO channel suffers from slow fading.

Since only the intensity modulation, direct detection laser communication systems are discussed here, the turbulence effect of concern is the intensity fluctuation of the laser beam traversing the atmosphere. The strength of the irradiance fluctuation in a turbulent medium is given by the variance of the log intensity, $l$ (also called the Roytov parameter $\sigma_{l} l^{2}$ ) and the transverse coherence length of a field travelling through a turbulent channel is denoted by $\rho_{\mathrm{o}}$. Over the range $l_{o} \leq \sqrt{\lambda L} \leq L_{o}$ these parameters are defined as (Osche, 2002):

$$
\begin{gathered}
\sigma_{l}^{2}=2.25(2 \pi / \lambda)^{7 / 6} \int_{0}^{\infty} C_{n}^{2}(x)(x / L)^{5 / 6}(L-x)^{5 / 6} d x \\
\rho_{o} \approx \sqrt{\lambda L}
\end{gathered}
$$


where $C_{n}{ }^{2}$ is the refractive index structure constant (which characterizes the strength of refractive index variation in the medium). A commonly used model for $C_{n}{ }^{2}$ is the HufnagelValley (H-V) model described by the following (Andrews et al., 2001):

$$
\begin{gathered}
C_{n}^{2}(h)=0.00594(v / 27)^{2}\left(10^{-5} h\right)^{10} \exp (-h / 1000)+2.7 \times 10^{-16} \exp (-h / 1500) \\
+\hat{A} \exp (-h / 100)
\end{gathered}
$$

$\hat{A}$ is taken as the nominal value of $C_{n}^{2}(0)$ at ground level in $\mathrm{m}^{-2 / 3}$. Generally, the structure parameter is assumed constant for a horizontal link and ranges from $10^{-15} \mathrm{~m}^{-2 / 3}$ for weak to $10^{-12} \mathrm{~m}^{-2 / 3}$ for strong turbulence regimes.

Considering single scattering characterized weak turbulence and assuming the log intensity $l$ of laser light traversing the turbulent atmosphere to be normally distributed, that is $l \sim N\left(-\sigma_{l}^{2} / 2, \sigma_{l}^{2}\right)$, then the probability density function (pdf) of the laser beam intensity, $I=I_{o} \exp (l)$, is given by:

$$
p(I)=\frac{1}{\sqrt{2 \pi \sigma_{l}^{2}}} \frac{1}{I} \exp \left\{-\frac{\left[\ln \left(I / I_{o}\right)+\sigma_{l}^{2} / 2\right]^{2}}{2 \sigma_{l}^{2}}\right\}
$$

where $I_{o}$ is the mean received intensity without turbulence. The normalised variance of the intensity $\sigma_{N}^{2}$ is derived as follows:

$$
\sigma_{N}^{2}=E\left[I^{2}\right]-(E[I])^{2} /(E[I])^{2}=\exp \left(\sigma_{l}^{2}\right)-1
$$

The turbulence model discussed thus far is the lognormal turbulence, it is only valid for the weak turbulence with $\sigma_{N}^{2}<1.2$. For $\sigma_{N}^{2} \geq 1.2$, saturation sets in and the model no longer holds. Turbulence induced irradiance fluctuation can enter saturation due to one or a combination of increased $C_{n}$, link length and reduced wavelength. Also, when multiple scatterings are experienced especially in longer link ranges, the incident wave becomes increasingly incoherent and log normal model becomes invalid. Though not discussed here, another model which has a wider range of validity but lacks the mathematical simplicity of lognormal is the gamma-gamma turbulence model. Moreover, in the limit of strong irradiance fluctuations (i.e. in saturation regime and beyond) where the link length spans several kilometres, the number of independent scatterings becomes large (Karp et al., 1988). In the saturation regime, irradiance fluctuation is believed to follow the negative exponential distribution.

\section{Noise Sources}

Background noise: This is due to radiations from both the sky (extended source) and the Sun (localised source). Background radiation from other celestial bodies such as stars and reflected background radiation are assumed too weak to be considered for terrestrial FSO links, they however contribute significantly to background noise in deep space FSO systems. 
The irradiance (power per unit area) expressions for both the extended and the localised background sources are given by the following equations:

$$
\begin{gathered}
I_{\text {sky }}=N(\lambda) \Delta \lambda \pi \Omega^{2} / 4 \\
I_{\text {sun }}=W(\lambda) \Delta \lambda
\end{gathered}
$$

where $N(\lambda)$ and $W(\lambda)$ are the spectral radiance of the sky and spectral radiant emittance of the Sun, respectively, $\Delta \lambda$ is the bandwidth of the optical BPF at the receiver, and $\Omega$ is the receiver FOV in radian. By carefully choosing a receiver with a very narrow FOV and $\Delta \lambda$, the impact of background noise can be greatly reduced. Optical BPF in the form of coatings on the receiver optics/telescope with $\Delta \lambda<1 \mathrm{~nm}$ are now readily available. Empirical values of $N(\lambda)$ and $W(\lambda)$ under different observation conditions are also available in reference (Gagliardi and Karp, 1995). The background noise is a shot noise and its variance is given by:

$$
\sigma_{B g}^{2}=2 q B \Re\left(I_{\text {sky }}+I_{\text {sun }}\right)
$$

where $B$ is the system electrical bandwidth and $\Re=\eta q \lambda / h c$ is the photodetector responsivity, $\eta$ is the detector quantum efficiency, $q$ is the electronic charge; $h$ and $c$ represent the Plank's constant and the speed of light in vacuum, respectively.

Quantum noise: A shot noise due to the statistical nature of the optical detection process. Its value is usually very small with variance:

$$
\sigma_{Q t m}^{2}=2 q B \Re I
$$

Thermal noise: This is the noise caused by the thermal fluctuations of electrons in a receiver circuit of equivalent resistance $R_{L}$, and temperature $T_{e}$. Its variance is given by:

$$
\sigma_{T h}^{2}=\frac{4 k T_{e} B}{R_{L}}
$$

The dark current and the relative intensity noise are usually so small and negligible. The total noise variance is thus given as:

$$
\sigma^{2}=\sigma_{Q t m}^{2}+\sigma_{B g}^{2}+\sigma_{T h}^{2}
$$


The major challenges associated with the optical wireless communication systems are summarised in Table 7.

\begin{tabular}{|c|c|c|c|c|}
\hline Challenge & Causes & Effects & Mitigation Approach & $\begin{array}{l}\text { Indoor } \\
\text { / } \\
\text { FSO }\end{array}$ \\
\hline \multirow{7}{*}{$\begin{array}{l}\text { Inter } \\
\text { symbol } \\
\text { interference } \\
\text { (ISI) }\end{array}$} & \multirow{7}{*}{$\begin{array}{l}\text { Multipath } \\
\text { Propagation }\end{array}$} & \multirow{2}{*}{$\begin{array}{l}\text { Poor transmission } \\
\text { quality (high BER) }\end{array}$} & Channel equalization & \multirow{7}{*}{ Indoor } \\
\hline & & & $\begin{array}{l}\text { Forward error control } \\
\text { (FEC) }\end{array}$ & \\
\hline & & \multirow{5}{*}{$\begin{array}{l}\text { Multipath } \\
\text { distortion or } \\
\text { dispersion } \\
\text { Reduced date rates }\end{array}$} & $\begin{array}{l}\text { Spread spectrum } \\
\text { techniques }\end{array}$ & \\
\hline & & & $\begin{array}{l}\text { Multiple subcarrier } \\
\text { Modulation (More } \\
\text { bandwidth efficient than a } \\
\text { single-carrier system) }\end{array}$ & \\
\hline & & & OFDM, MSM & \\
\hline & & & Multi-beam transmitter & \\
\hline & & & FOV controlling & \\
\hline \multirow[t]{2}{*}{ Safety } & \multirow[t]{2}{*}{$\begin{array}{l}\text { Laser } \\
\text { Radiation }\end{array}$} & \multirow[t]{2}{*}{$\begin{array}{l}\text { Damage to eyes } \\
\text { and skin }\end{array}$} & $\begin{array}{l}\text { Power efficient } \\
\text { modulation schemes: - } \\
\text { PPM, DPIM, etc. }\end{array}$ & \multirow[t]{2}{*}{ Both } \\
\hline & & & $\begin{array}{l}\text { Use LED, Class } 1 \text { lasers, } \\
\text { and } 1550 \mathrm{~nm} \text { wavelength }\end{array}$ & \\
\hline \multirow{7}{*}{ Noise } & $\begin{array}{l}\text { Dark current } \\
\text { noise }\end{array}$ & \multirow{7}{*}{$\begin{array}{l}\text { Low Signal-to-noise } \\
\text { ratio and high BER }\end{array}$} & \multirow{5}{*}{$\begin{array}{l}\text { Optical and electrical } \\
\text { filtering } \\
\text { Pre-amplification } \\
\text { FEC } \\
\text { Low noise post detection } \\
\text { amplifier }\end{array}$} & \multirow{7}{*}{ Both } \\
\hline & Shot noise & & & \\
\hline & $\begin{array}{l}\text { Background } \\
\text { noise }\end{array}$ & & & \\
\hline & $\begin{array}{l}\text { Thermal } \\
\text { noise }\end{array}$ & & & \\
\hline & $\begin{array}{l}\text { Relative } \\
\text { intensity } \\
\text { noise }\end{array}$ & & & \\
\hline & $\begin{array}{l}\text { Excess noise } \\
\text { (with APD) }\end{array}$ & & \multirow{2}{*}{$\begin{array}{l}\text { Small FOV lasers } \\
\text { Optical filter }\end{array}$} & \\
\hline & $\begin{array}{l}\text { ASE (only if } \\
\text { optical } \\
\text { amplifier is } \\
\text { used) }\end{array}$ & & & \\
\hline \multirow{3}{*}{ Turbulence } & \multirow{3}{*}{$\begin{array}{l}\text { Random } \\
\text { refractive } \\
\text { index } \\
\text { variation }\end{array}$} & $\begin{array}{l}\text { Phase and intensity } \\
\text { fluctuations } \\
\text { (scintillation) }\end{array}$ & \multirow{3}{*}{$\begin{array}{l}\text { FEC (LDPC, Turbo codes) } \\
\text { Robust modulation: SIM, } \\
\text { PPM } \\
\text { MIMO } \\
\text { Diversity reception } \\
\text { (temporal and spatial) }\end{array}$} & \multirow{3}{*}{ FSO } \\
\hline & & Image dancing & & \\
\hline & & $\begin{array}{l}\text { Spatial coherence } \\
\text { degradation }\end{array}$ & & \\
\hline
\end{tabular}




\begin{tabular}{|c|c|c|c|c|}
\hline & & Beam spreading & Adaptive optics & \\
\hline $\begin{array}{l}\text { Reflection } \\
\text { index }\end{array}$ & $\begin{array}{l}\text { Different } \\
\text { materials }\end{array}$ & $\begin{array}{l}\text { Higher losses due } \\
\text { to reflection on } \\
\text { surfaces }\end{array}$ & Higher transmit power & Indoor \\
\hline \multirow{4}{*}{ Blocking } & Furniture & \multirow{4}{*}{$\begin{array}{l}\text { Temporary link } \\
\text { outage }\end{array}$} & Diffuse link & \multirow{4}{*}{ Both } \\
\hline & $\begin{array}{l}\text { Moving } \\
\text { objects }\end{array}$ & & Cellular system & \\
\hline & Walls & & Multi-beam & \\
\hline & Birds & & Hybrid FSO/RF & \\
\hline $\begin{array}{l}\text { Weather } \\
\text { effects }\end{array}$ & $\begin{array}{l}\text { Fog, Rain, } \\
\text { Gases, } \\
\text { Smoke, } \\
\text { Aerosols }\end{array}$ & $\begin{array}{l}\text { Attenuations, } \\
\text { Scattering } \\
\text { Link outage }\end{array}$ & $\begin{array}{l}\text { Higher transmit power } \\
\text { Hybrid FSO/RF }\end{array}$ & FSO \\
\hline $\begin{array}{l}\text { Pointing, } \\
\text { Acquisition } \\
\text { and } \\
\text { Tracking } \\
\text { (PAT) } \\
\end{array}$ & $\begin{array}{l}\text { Mobile link } \\
\text { heads } \\
\text { Building } \\
\text { sway }\end{array}$ & $\begin{array}{l}\text { Temporary/perma } \\
\text { nent link outage } \\
\text { Power loss }\end{array}$ & $\begin{array}{l}\text { Hybrid FSO/RF } \\
\text { Active tracking Adaptive } \\
\text { optics (beam steering and } \\
\text { tracking) }\end{array}$ & FSO \\
\hline
\end{tabular}

Table 7. Challenges in Optical Wireless Communications

\section{Modulation Techniques}

There exist different types of modulation schemes that are suitable for optical wireless communication systems, one of such is the family of pulse modulation techniques shown in Fig. 10. Since the average emitted optical power is limited, the different modulation techniques are usually compared in terms of the average received optical power required to achieve a desired bit error rate at a given data rate. A power efficient modulation scheme is desirable in order to maximise the ratio of peak to average power. Here, the performance analysis of an FSO system based on the following modulation techniques: On-off keying (OOK), pulse position modulation (PPM) and subcarrier intensity modulation (SIM) for non-ideal channels will be highlighted. 


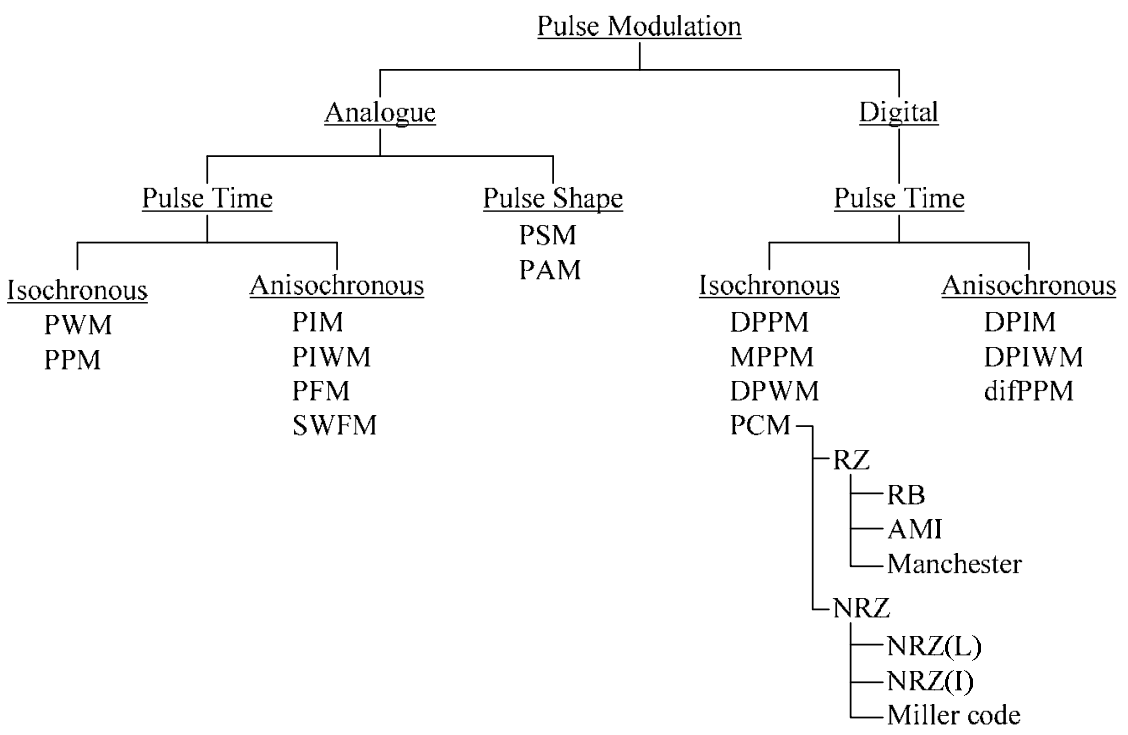

Fig. 10. Pulse modulation tree.

\subsection{On-Off Keying}

The OOK signalling is the dominant modulation scheme employed in terrestrial wireless optical communication systems. This is primarily due to its simplicity and resilience to laser nonlinearity. OOK can use either non-return-to-zero (NRZ) or return-to-zero (RZ) pulse formats. In NRZ-OOK, an optical pulse of peak power $\mathrm{a}_{e} P_{T}$ represents a digital symbol ' 0 ' while the transmission of an optical pulse of peak power $P_{T}$ represents a digital symbol ' 1 '. The optical source extinction ratio $\alpha_{\mathrm{e}}$ lies in the range $0 \leq \alpha_{\mathrm{e}}<1$. The finite duration of the optical pulse is the same as the symbol duration T. With OOK-RZ, the pulse duration is lower than the bit duration, giving an improvement in power efficiency over NRZ-OOK at the expense of an increased bandwidth requirement. Without any loss of generality, the receiver area can be normalised to unity such that the optical power can henceforth be represented by the optical intensity $I$. If $\mathfrak{R}$ represents the responsivity of the PIN photodetector, the received signal in an OOK modulated FSO system becomes:

$$
i(t)=\Re\left[\left[1+\sum_{j=-\infty}^{\infty} d_{j} g(t-j T)\right]+n(t)\right.
$$

where $n(t) \sim N\left(0, \sigma^{2}\right)$ is the additive white Gaussian noise and $d_{j}=[-1,0]$. In all the analyses that follow, the extinction ratio is assumed equal to zero unless otherwise stated. At the receiver, the received signal is fed into a threshold detector which compares the received signal with a pre-determined threshold level. A digital symbol ' 11 ' is assumed received if the received signal is above the threshold level and ' 0 ' otherwise. The probability of error is therefore given as: 


$$
P_{e}=p(0) \int_{i_{t h}}^{\infty} p(i / 0) d i+p(1) \int_{0}^{i_{t h}} p(i / 1) d i
$$

where the marginal probabilities are defined as:

$$
\begin{gathered}
p(i / 0)=\frac{1}{\sqrt{2 \pi \sigma^{2}}} \exp \left(-i^{2} / 2 \sigma^{2}\right) \\
p(i / 1)=\frac{1}{\sqrt{2 \pi \sigma^{2}}} \exp \left[\frac{-(i-\Re I)^{2}}{2 \sigma^{2}}\right]
\end{gathered}
$$

For equiprobable symbols, $p(0)=p(1)=0.5$, the optimum threshold point is at $i_{t h}=0.5 \Re I$. And the conditional probability of error reduces to:

$$
P_{e c}=Q\left(\frac{i_{t h}}{\sigma}\right)
$$

where $\mathrm{Q}(x)=0.5 \operatorname{erfc}(\mathrm{x} / \sqrt{2})$. But in the presence of atmospheric turbulence, the threshold level is no longer fixed midway between the signal levels representing symbols ' 1 ' and ' 0 '. The marginal probability $p(i / 1)$ is then modified by averaging equation (32) over the scintillation statistics to arrive at equation (34). Note that scintillation does not occur when no pulse is transmitted.

$$
p(i / 1)=\int_{0}^{\infty} p(i / 1, I) p(I) d I
$$

Assuming equiprobable symbol transmission and invoking the maximum a posteriori symbol-by-symbol detection, the likelihood function becomes (Popoola et al., 2008):

$$
\Lambda=\int_{0}^{\infty} \exp \left[\frac{-(i-\Re I)^{2}-i^{2}}{2 \sigma^{2}}\right] p(I) d I
$$

The threshold level $i_{\text {th }}$ is obtained from (35) with $\Lambda=1$. Based on the log normal turbulence model, the plot of $i_{\text {th }}$ for different levels of turbulence is shown in Fig. 11. 


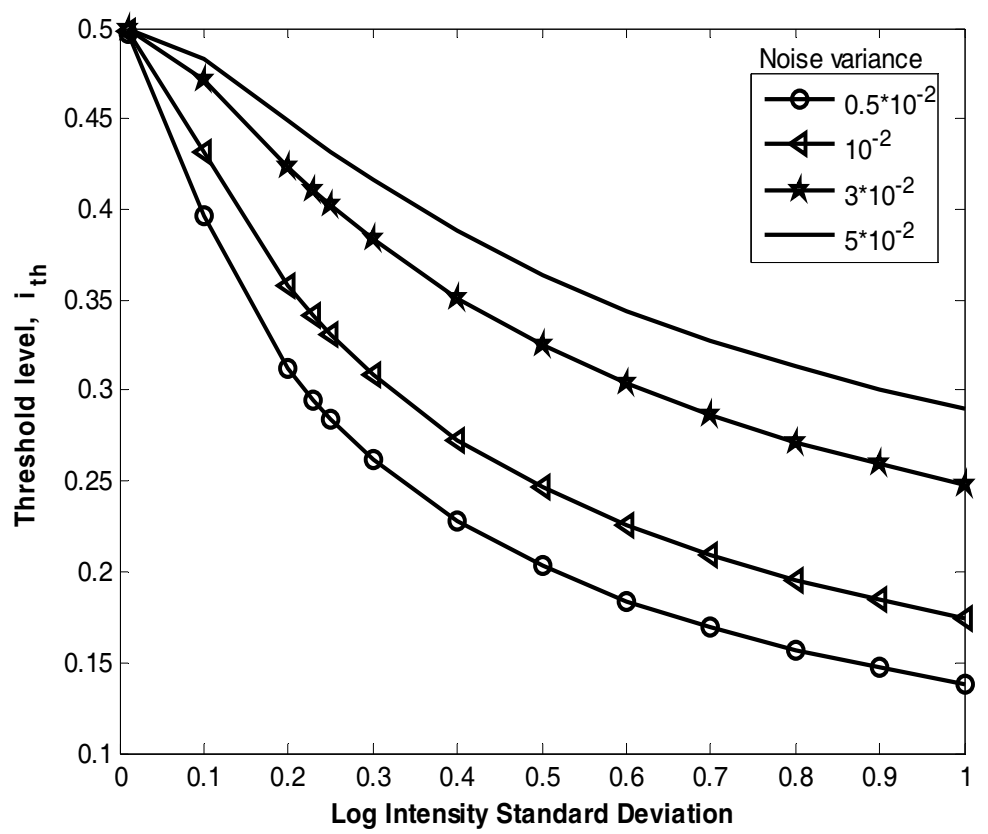

Fig. 11. OOK threshold level against the log intensity standard deviation for a range of turbulence levels.

The threshold level is observed to approach the 0.5 value as the scintillation level approaches zero. As an illustration, at a turbulence level $\sigma_{l}{ }^{2}=0.2$, the probability of bit error $P_{e}$, obtained from the combination of (30), (31) and (34) is plotted against the normalised SNR $=(\Re E[I])^{2} / \sigma^{2}$ in Fig. 12, the value of $i_{t h}$ used for the adaptive threshold level graph is obtained from the solution of equation (35). From this figure, the effect of using a fixed threshold level in fading channels results in a BER floor. The values of which depend on the fixed threshold level and turbulence induced fading strength. With an adaptive threshold, there is no such BER floor and any desired level of BER can thus be realised. 


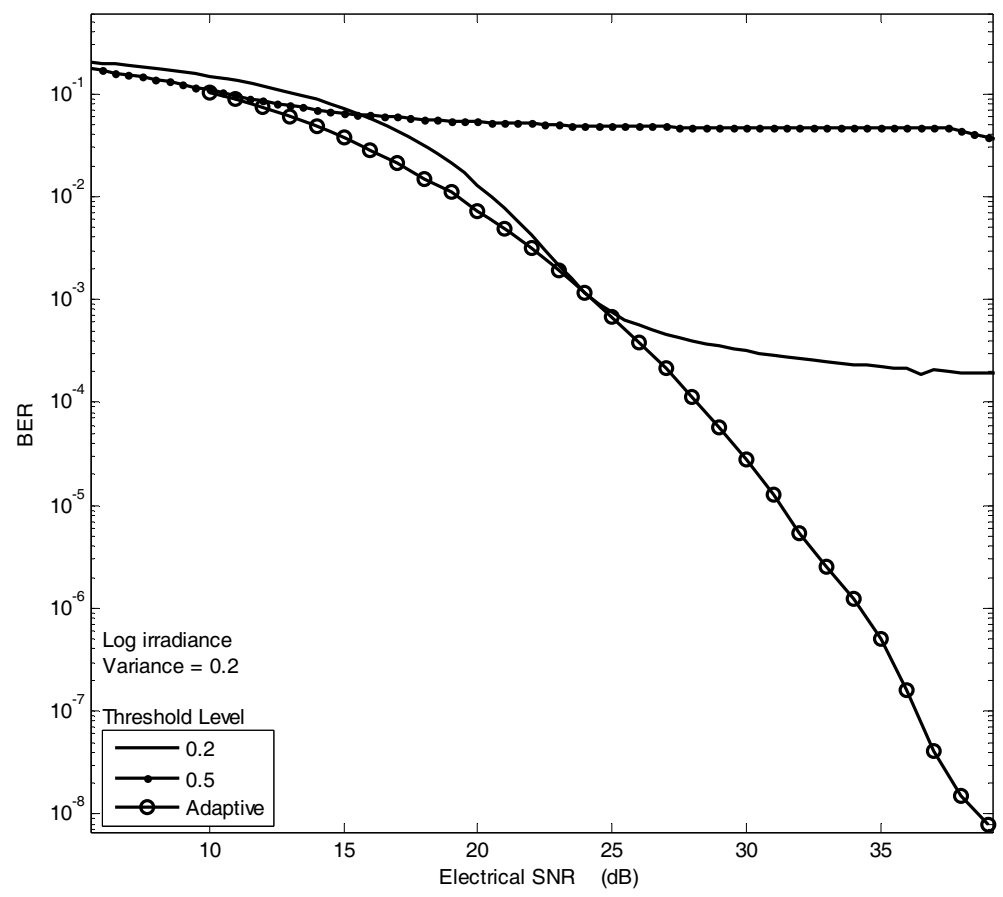

Fig. 12. BER performance of OOK based FSO in atmospheric turbulence, $\sigma_{l}^{2}=0.2$.

In Fig. 13, the BER is again plotted against the normalised SNR at various levels of scintillation including when the threshold is fixed at 0.5 . This is intended to show the effect of turbulence strength on the amount of SNR required to maintain a given error performance level. With a fixed threshold, the BER reaches a floor at a BER that is greater than $10^{-4}$, meaning that a lower BER is not achievable at the specified low scintillation level. From this graph, it can be inferred that atmospheric turbulence: i) causes SNR penalty, for example $\sim 26 \mathrm{~dB}$ of SNR is needed to achieve a BER of $10^{-6}$ due to a very weak scintillation of strength $\sigma_{l}{ }^{2}=0.252$, this however increases by over $20 \mathrm{~dB}$ as the scintillation strength increases to $\sigma_{l}^{2}=0.72$; and ii) implies that adaptive threshold will be required to avoid a BER floor in the system performance. 


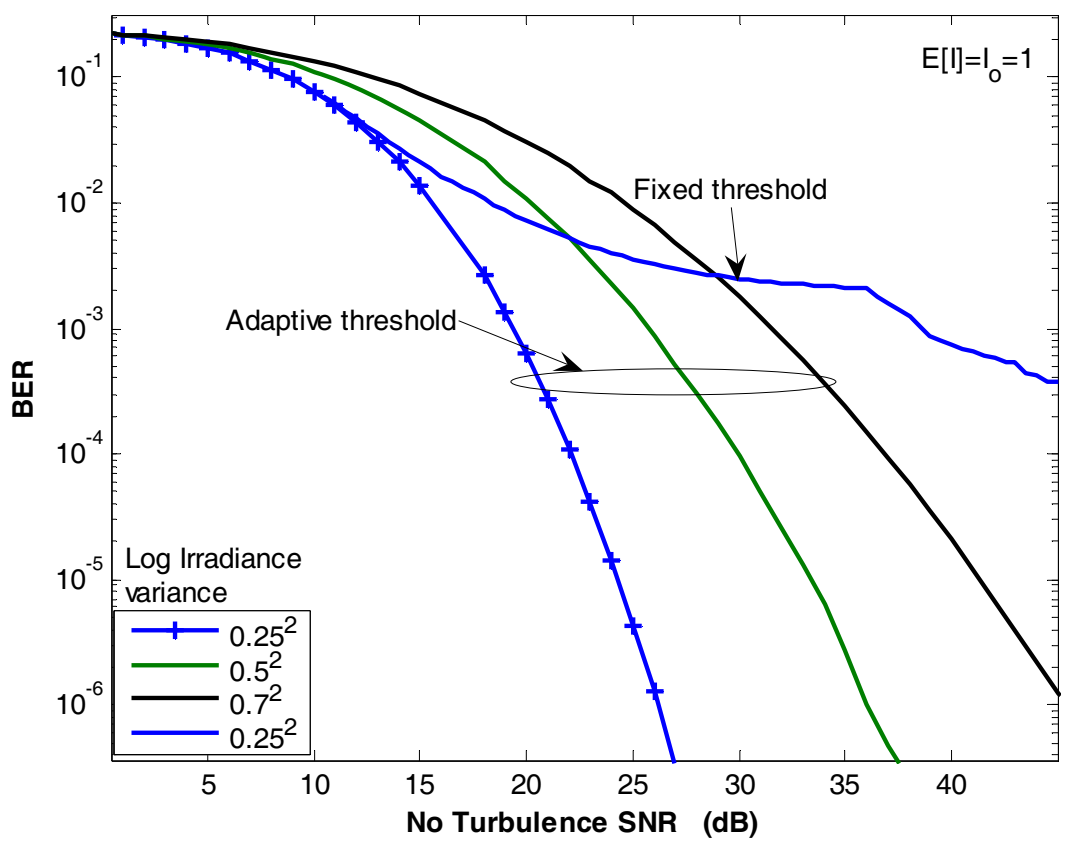

Fig. 13. BER against the SNR for fixed and adaptive thresholds and various levels of scintillation.

The results of this section illustrate that for the OOK modulated FSO system to perform at its best, the receiver will require knowledge of both the fading strength and the noise level. This can be resolved by integrating into the system an intensity estimation network which can predict the scintillation level based on past events. The implementation of this is not trivial, and as such, commercial FSO designers tend to adopt the fixed threshold approach and include a sufficiently large link margin in the link budget to cater for turbulence induced fading.

\subsection{Pulse Position Modulation}

This is an orthogonal modulation technique and a member of the pulse modulation family (see Fig. 10). The PPM modulation technique improves on the power efficiency of OOK but at the expense of an increased bandwidth requirement and greater complexity. In PPM, each block of $\log _{2} M$ data bits is mapped to one of $M$ possible symbols. Generally, the notation $M$ PPM is used to indicate the order. Each symbol consists of a pulse of constant power $P_{T}$ occupying one slot, along with $M-1$ empty slots. The position of the pulse corresponds to the decimal value of the $\log _{2} M$ data bits. Hence, the information is encoded by the position of the pulse within the symbol. The slot duration $T_{s}$ is related to the bit duration by the following expression: 


$$
T_{S}=\frac{T \log _{2} M}{M}
$$

The transmitted waveforms for 16-PPM and OOK are shown in Fig. 14.

$M$

Fig. 14. Transmitted waveforms for OOK and 16-PPM

A PPM receiver will require both slot and symbol synchronisation in order to demodulate the information encoded on the pulse position. Nevertheless, because of its superior power efficiency, PPM is an attractive modulation technique for optical wireless communication systems particularly in deep space laser communication applications. Assuming that a complete synchronisation is maintained between the transmitter and receiver at all times, the optical receiver detects the transmitted signal by attempting to determine the optical energy in each possible time slot. In direct photodetection this is equivalent to "counting" the number of released electrons in each $T_{s}$ interval. The photo count per PPM slot is given by:

$$
K_{S}=\frac{\eta \lambda P_{R} T_{S}}{h c}
$$

where $P_{R}$ is the received optical power during a slot duration. An APD could be used to afford an increase in the amount of photon count per PPM slot but unfortunately, the photomultiplication process that governs the generation of the secondary electrons is a random process. This implies that a large photo-multiplication gain will eventually lead to a large noise factor and an error prone performance. For a moderately high received signal as is the case in commercial and short range FSO systems, the BER conditioned on $K_{s}$ is given by (Gagliardi and Karp, 1995): 


$$
P_{e c}=Q\left(\sqrt{\frac{(\bar{g} q)^{2} K_{s}^{2}}{(\bar{g} q)^{2} F\left(K_{s}+2 K_{B g}\right)+2 \sigma_{T h}^{2}}}\right)
$$

where the parameters are defined as:

\begin{tabular}{|l|l|}
\hline$K_{B g}=\eta \lambda P_{B g} T_{S} / h c$ & $\begin{array}{l}\text { Average photon count per PPM slot due to the } \\
\text { background Radiation of power } P_{B g}\end{array}$ \\
\hline $\bar{g}$ & Average APD gain \\
\hline$q$ & Electronic charge \\
\hline$F \approx 2+\zeta \bar{g}$ & Noise factor of the APD \\
\hline$\zeta$ & APD ionisation factor \\
\hline$\sigma_{T h}^{2}=\left(2 \kappa T_{e} q / R_{L}\right)\left(T_{S}\right)$ & $\begin{array}{l}\text { Equivalent thermal noise count within a PPM } \\
\text { slot duration. }\end{array}$ \\
\hline$R_{b}=1 / T$ & Bit rate. \\
\hline$\kappa$ & Boltzmann's constant \\
\hline$R_{L}$ & Equivalent load resistance \\
\hline
\end{tabular}

In the presence of log normal atmospheric turbulence, the unconditional BER for a binary PPM modulated FSO obtained by averaging (38) over the scintillation statistics can be approximated as (Kiasaleh, 2005):

$$
P_{e} \approx \frac{1}{\sqrt{\pi}} \sum_{i=1}^{n} w_{i} \mathrm{Q}\left(\frac{\exp \left(2\left(\sqrt{2} \sigma_{k} x_{i}+m_{k}\right)\right)}{F \exp \left(\sqrt{2} \sigma_{k} x_{i}+m_{k}\right)+K_{n}}\right)
$$

where $\left[w_{i}\right]_{i=1}^{n}$ and $\left[x_{i}\right]_{i=1}^{n}$ are the weight factors and the zeros of an $n^{\text {th }}$ order Hermite polynomial. $K_{n}=\left(2 \sigma_{T h}^{2} /(\bar{g} q)^{2}\right)+2 F K_{B g}$ and $\sigma_{k}^{2}=\ln \left(\sigma_{N}^{2}+1\right)$. It is noteworthy that the fluctuation of the mean count $K_{s}$ is brought about by the atmospheric turbulence and its ensemble average is given by:

$$
E\left[K_{s}\right]=\exp \left(\frac{\sigma_{k}^{2}}{2}+m_{k}\right)
$$

For an M-PPM system, the BER denoted by $P_{e}^{M}$ has an upper bound given by (Kiasaleh, 2005): 


$$
P_{e}^{M} \leq \frac{M}{2 \sqrt{\pi}} \sum_{i=1}^{n} w_{i} \mathrm{Q}\left(\frac{\exp \left(2\left(\sqrt{2} \sigma_{k} x_{i}+m_{k}\right)\right)}{F \exp \left(\sqrt{2} \sigma_{k} x_{i}+m_{k}\right)+K_{n}}\right)
$$

The BER of a binary PPM modulated FSO is shown in Fig. 15 for different levels of scintillation.

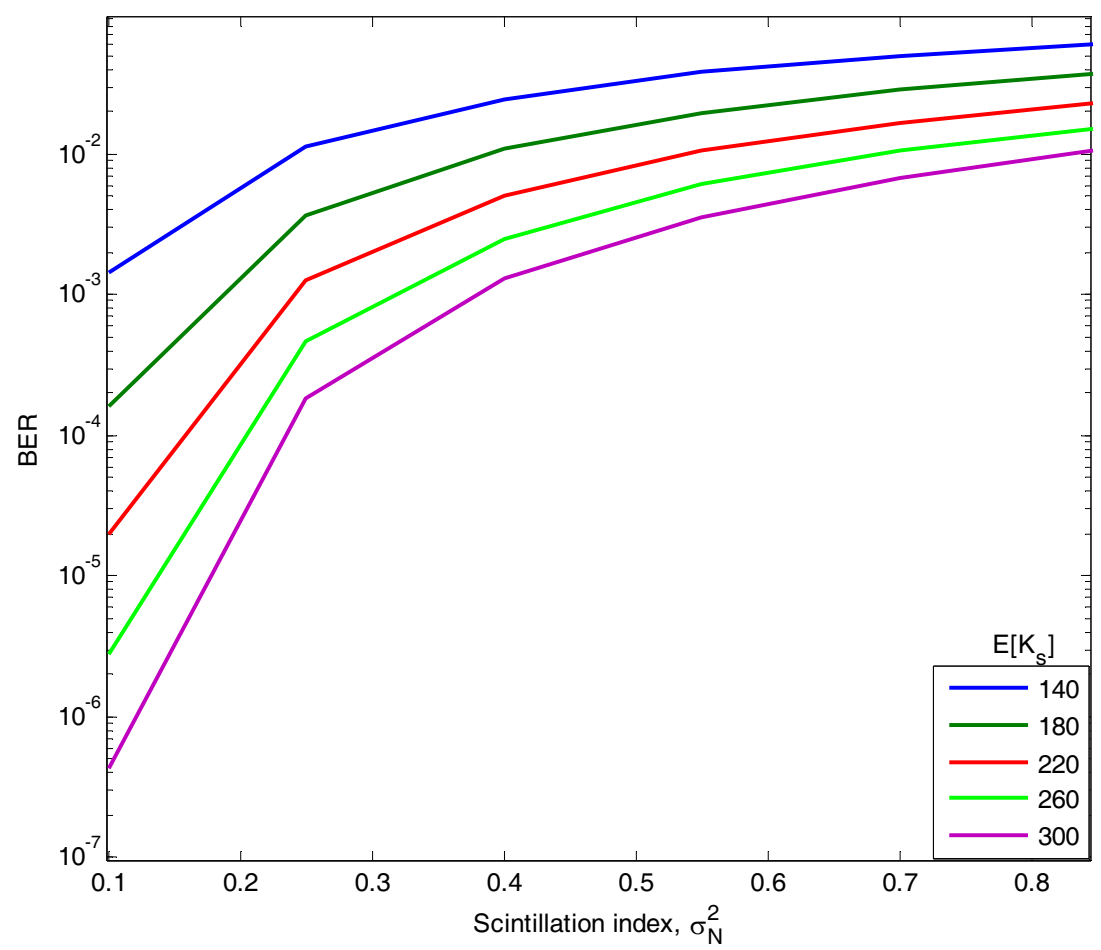

Fig. 15. Binary PPM BER as a function of scintillation index for $K_{B g}=10 ; T_{e}=300 \mathrm{~K}, \zeta=0.028$, $R_{b}=155 \mathrm{Mbps}$ and $\bar{g}=150$.

As expected, an increase in the atmospheric scintillation results in an increase in the required signal level to achieve a given BER. Increasing the signal strength can be used to minimise the scintillation effect at a low scintillation index, but as turbulence strength increases, it is observed that the BERs tend towards a high asymptotic value. 


\subsection{Subcarrier Intensity Modulation}

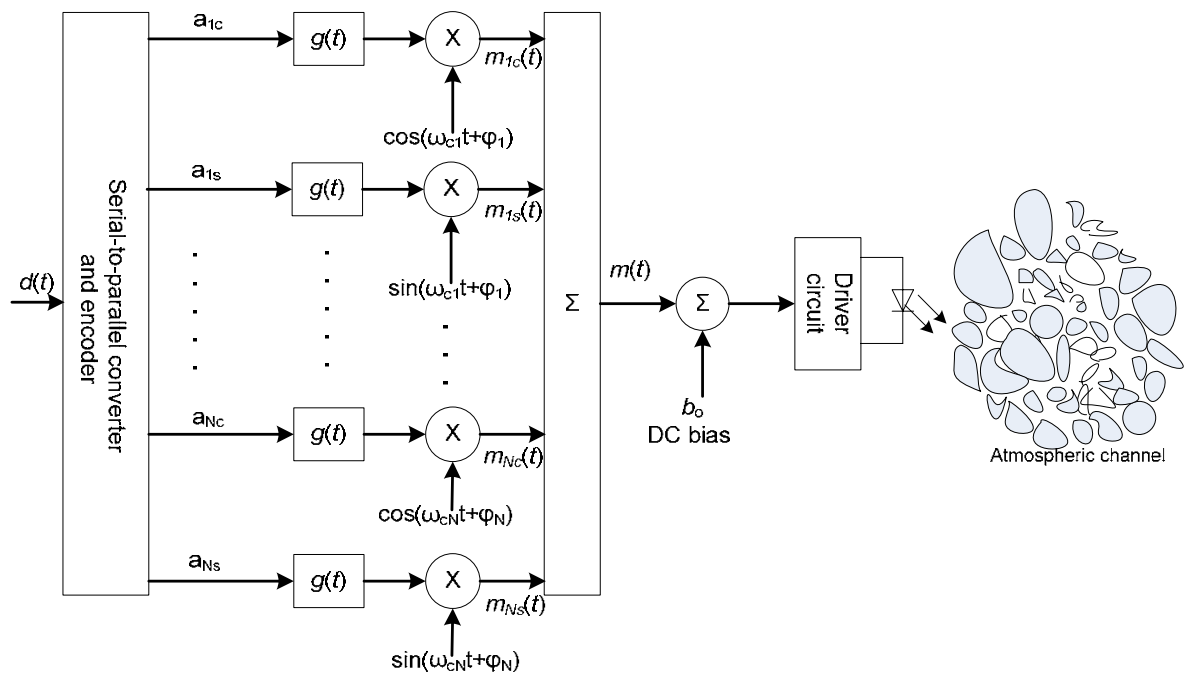

(a)

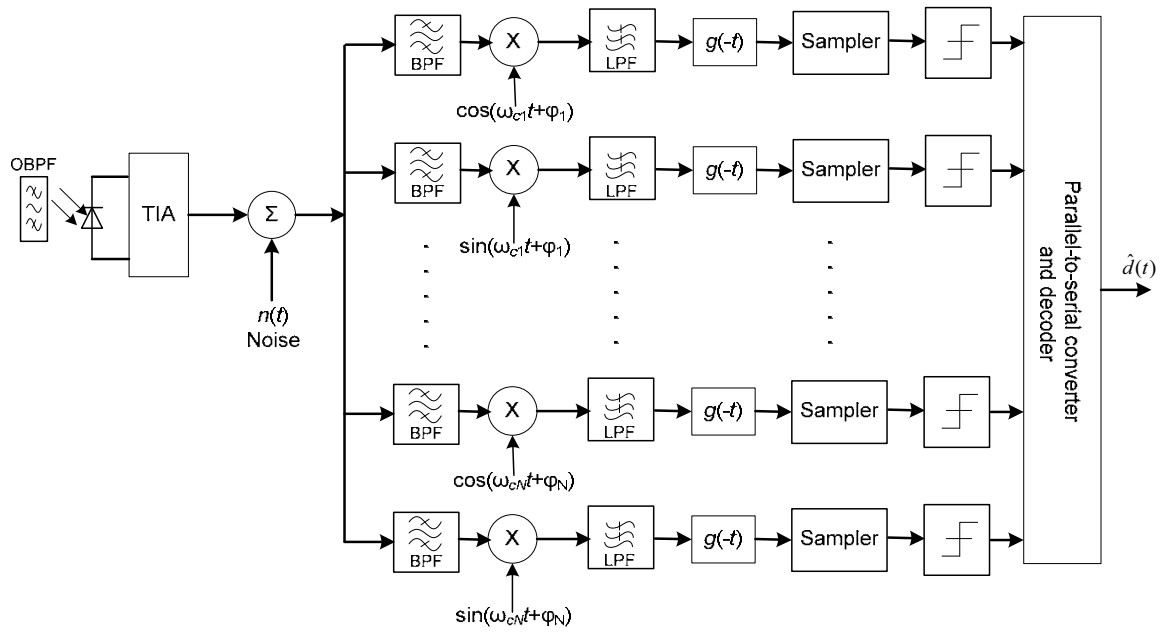

(b)

Fig. 16. Block diagram of SIM: (a) transmitter, and (b) receiver. TIA- trans-impedance

In optical SIM, an RF subcarrier signal pre-modulated with the source data $d(t)$ is used to modulate the intensity of the optical carrier - a continuous wave laser diode (Popoola and Ghassemlooy, 2009). Figure 16 illustrates the system block diagram of a SIM-FSO with $N$ subcarriers. The serial-to-parallel converter distributes the incoming data across the $N$ subcarriers. Each subcarrier carries a reduced symbol rate but the aggregate must be equal to the symbol rate of $d(t)$. Another obvious possibility not shown in the figure is to have 
different users occupying the $N$ different subcarriers. Prior to modulating the laser irradiance, the source data $d(t)$ is modulated onto the RF subcarriers. For the M-PSK subcarrier modulation shown in Fig. 16, the encoder maps each subcarrier symbol onto the symbol amplitude $\left\{a_{i c}, a_{i s}\right\}_{i=1}^{N}$ that corresponds to the constellation in use. Since the subcarrier signal $m(t)$ is sinusoidal having both positive and negative values, a DC level $b_{0}$ is added to $m(t)$ before it is used to directly drive the laser diode. This is to ensure that the bias current is always equal to or greater than the threshold current - a prerequisite for the avoidance of signal clipping.

The following gives the general expression for $m(t)$ in the N-SIM-FSO system:

$$
m(t)=\sum_{i=1}^{N} m_{i}(t)
$$

During symbol duration, each RF subcarrier signal is generally represented by:

$$
m_{i}(t)=g(t) a_{i c} \cos \left(\omega_{c i} t+\varphi_{i}\right)+g(t) a_{i s} \sin \left(\omega_{c i} t+\varphi_{i}\right)
$$

where $g(t)$ is the pulse shaping function, the subcarrier angular frequency and phase are represented by $\left[\omega_{c i}, \varphi_{i}\right]_{i=1}^{N}$. It follows that each subcarrier can be modulated by any standard RF digital/analogue modulation techniques such as QAM, M-PSK, M-FSK and M-ASK. SIM benefits from advances made in RF modulators/demodulators, stable oscillators and narrow band filters.

Using a direct detection at the receiver, the incoming optical radiation is converted into an electrical signal. This is followed by a standard RF demodulator to recover the transmitted symbol as shown in Fig. 16(b). The received signal is modelled as:

$$
i(t)=\Re I[1+\xi m(t)]+n(t)
$$

where $\xi$ is the modulation depth/index. The electrical band pass filters (BPF) performs the following functions: selection of the individual subcarrier for demodulation, reduction of the noise power and suppression of any slow varying $R I$ component present in the received signal. For a subcarrier at $\omega_{c i}$ the received signal is:

$$
i(t)=I_{c o m p}+Q_{c o m p}
$$

where

$$
\begin{gathered}
I_{\text {comp }}=\Re I \xi g(t) a_{i c} \cos \left(\omega_{c i} t+\varphi_{i}\right)+n_{I}(t) \\
Q_{c o m p}=-\Re I \xi g(t) a_{i s} \sin \left(\omega_{c i} t+\varphi_{i}\right)+n_{Q}(t)
\end{gathered}
$$

$n_{I}(t)$ and $n_{Q}(t)$ are the independent AWGN with zero mean and variance $\sigma^{2}$. By down converting the quadrature components $I_{\text {comp }}$ and $Q_{\text {comp }}$ by the reference signals $\cos \omega_{c} t$ and 
$\sin \omega_{c} t$, respectively, the following conditional BER expressions (47a-d) are obtained (Proakis, 2004):

$$
\begin{array}{cl}
P_{e c}=\mathrm{Q}(\sqrt{\gamma(I)}) & \text { for BPSK } \\
P_{e c} \approx \frac{2}{\log _{2} M} \mathrm{Q}\left(\sqrt{\left(\log _{2} M\right) \gamma(I)} \sin (\pi / M)\right) & \text { for M-PSK, } M \geq 4 \\
P_{e c}=0.5 \exp (-0.5 \gamma(I)) & \text { For DPSK } \\
P_{e c}=\frac{2(1-1 / \sqrt{M}}{\log _{2} M} \mathrm{Q}\left(\sqrt{\frac{3 \log _{2} M \gamma(I)}{2(M-1)}}\right) & \text { for M-QAM, } \log _{2} M \text { even }
\end{array}
$$

The SNR per bit and the average power are respectively given as:

$$
\begin{gathered}
\gamma(I)=\frac{(\xi \Re I)^{2} P_{m}}{\sigma^{2}} \\
P_{m}=\frac{A^{2}}{2 T} \int_{0}^{T} g^{2}(t) d t
\end{gathered}
$$

To illustrate the system performance in atmospheric turbulence, the BPSK-SIM is considered. Since each subcarrier is demodulated separately and independently, the unconditional BER per subcarrier is obtained by averaging (47a) over the irradiance fluctuation statistics to obtain:

$$
\begin{gathered}
P_{e}=\int_{0}^{\infty} \mathrm{Q}(\sqrt{\gamma(I)}) p(I) d I \\
P_{e}=\frac{1}{\sqrt{\pi}} \sum_{i=1}^{n} w_{i} \mathrm{Q}\left[\sqrt{K_{0}} \exp \left(K_{1}\left(\sqrt{2} \sigma_{l} x_{i}-\sigma_{l}^{2} / 2\right)\right)\right]
\end{gathered}
$$

where $\left[w_{i}\right]_{i=1}^{n}$ and $\left[x_{i}\right]_{i=1}^{n}$ are the weight factors and the zeros of an $n^{\text {th }}$ order Hermite polynomial. The values of $K_{1}$ and $K_{0}$ are given in Table 8 for different noise limiting conditions:

\begin{tabular}{|c|cccc|}
\cline { 2 - 5 } \multicolumn{1}{c|}{} & \multicolumn{4}{c|}{ Performance Limiting conditions } \\
\cline { 2 - 5 } \multicolumn{1}{c|}{} & Quantum limit & Thermal noise & Background noise & $\begin{array}{l}\text { Thermal and } \\
\text { background noise }\end{array}$ \\
\hline$K_{0}$ & $\frac{\xi^{2} \Re I_{o} P_{m}}{2 q R_{b}}$ & $\frac{\left(\xi \Re I_{o}\right)^{2} P_{m} R_{L}}{4 k T_{e} R_{b}}$ & $\frac{\left(\xi I_{o}\right)^{2} \Re P_{m}}{2 q R_{b}\left(I_{\text {sky }}+I_{\text {sun }}\right)}$ & $\frac{\left(\xi \Re I_{o}\right)^{2} P_{m}}{\left(\sigma_{B g}^{2}+\sigma_{T h}^{2}\right)}$ \\
$K_{1}$ & 0.5 & 1 & 1 & 1 \\
\hline
\end{tabular}

Table 8 . Values of $K_{1}$ and $K_{0}$ shown for different noise limiting conditions 
To keep the continuous wave laser source within its dynamic range, the condition $|\xi m(t)| \leq$ 1 must always hold. This condition places an upper bound on the amplitude of each subcarrier for a given value of $\xi$. It also implies that the use of multi-user-SIM (subcarrier multiplexing) incurs a $20 \log N \mathrm{~dB}$ reduction in $\gamma(I)$. Consequently, multiple subcarriers should only be considered when the need for increased capacity outweighs the accompanying power penalty.

Based on the simulation parameters of Table 9, the BER defined in (51) is plotted against the receiver sensitivity in Fig. 17 with $\xi=N=1$ under the different noise conditions. The figure illustrates clearly that for an FSO link with a suitable optical BPF and a narrow FOV detector, the system performance is limited by thermal noise. Moreover, under this thermal noise limited condition, the SIM-FSO still requires about $30 \mathrm{~dB}$ of SNR more, compared with the theoretical quantum limit.

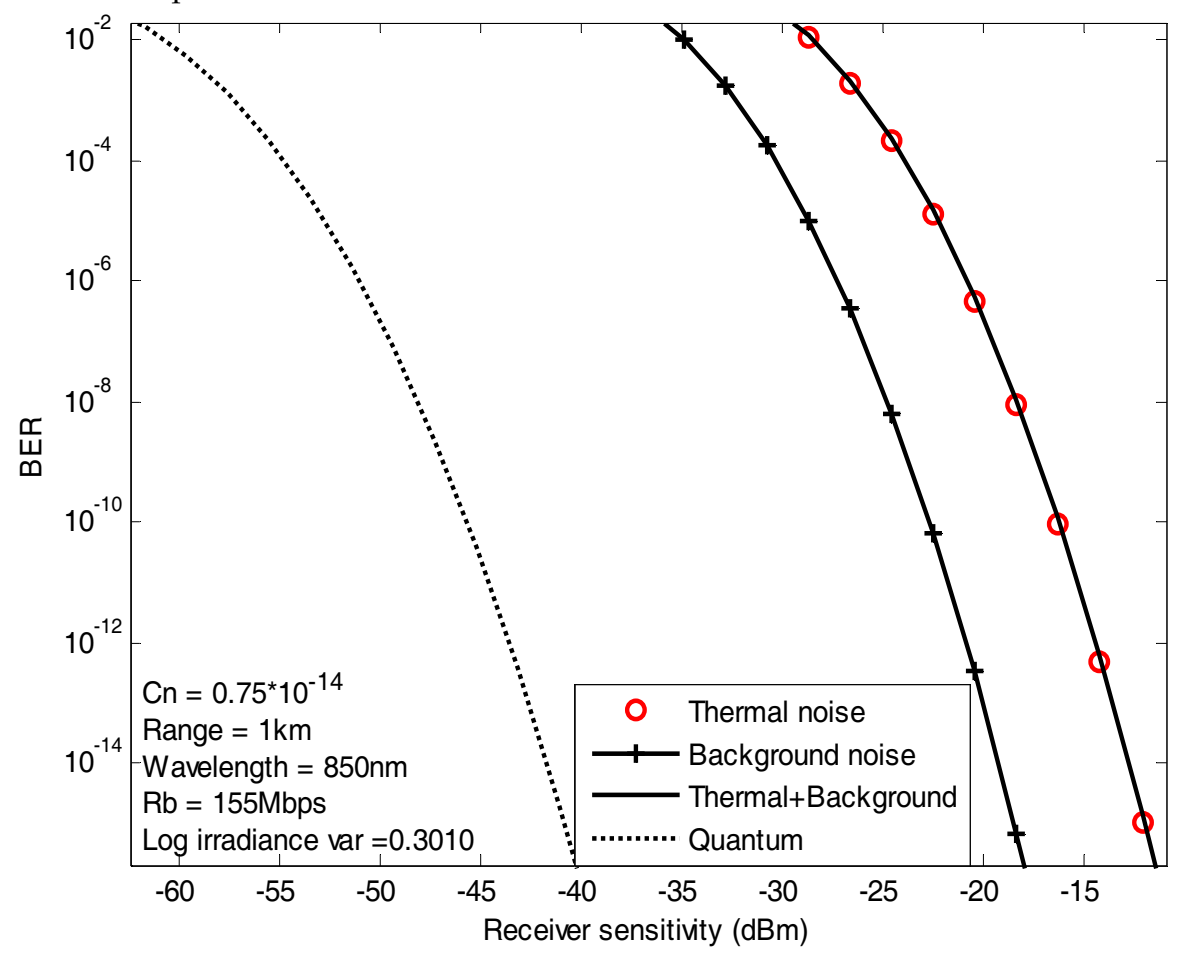

Fig. 17. The BER against the receiver sensitivity under different noise sources, with $R_{b}=155$ Mpbs, $\sigma_{\mathrm{l}}^{2}=0.3$ and $\xi=1$. 


\begin{tabular}{|ll|}
\hline Parameter & Value \\
\hline Symbol rate $R_{b}$ & $155 \mathrm{Mbps}$ \\
Spectral radiance of the sky $N(\lambda)$ & $10^{-3} \mathrm{~W} / \mathrm{cm}^{2} \mu \mathrm{mSr}$ \\
Spectral radiant emittance of the sun $W(\lambda)$ & $0.055 \mathrm{~W} / \mathrm{cm}^{2} \mu \mathrm{m}$ \\
Optical band-pass filter bandwidth $\Delta \lambda @ \lambda=850 \mathrm{~nm}$ & $1 \mathrm{~nm}$ \\
PIN photodetector field of view (FOV) & $0.6 \mathrm{rad}$ \\
Radiation wavelength $\lambda$ & $850 \mathrm{~nm}$ \\
Number of subcarriers $N$ & 1 \\
Link range $L$ & $1 \mathrm{~km}$ \\
Index of refraction structure parameter $C_{n}{ }^{2}$ & $0.75 \mathrm{~m}^{-2 / 3}$ \\
Load resistance $R_{L}$ & $50 \Omega$ \\
PIN photodetector responsivity $R$ & 1 \\
Operating temperature $T_{e}$ & $300 \mathrm{~K}$ \\
\hline
\end{tabular}

Table 9. Simulation parameters

\section{Conclusion}

In this chapter, the terrestrial FSO communication system has been discussed right from its basics to error performance based on OOK, PPM and SIM modulation schemes. The properties of the atmospheric channel have also been highlighted in terms of signal attenuation and scintillation. On the evidence of increasing deployment of FSO systems for civil applications, it is fair to say that the technology has now moved on from being researchers' pastime and bread board technology into a potent complementary access network technology. The provision of long range FSO links covering several kilometres with $99.999 \%$ availability across all weather conditions however remains a daunting challenge.

\section{References}

AFGL (1986) AFGL Atmospheric Constituent Profiles (0-120 km), AFGL-TR-86-0110, Air Force Geophysics Laboratory, Hanscom Air Force Base, Massachusetts,.

AL NABOULSI, M. \& SIZUN, H. (2004) Fog attenuation prediction for optical and infrared waves. Optical Engineering, 23, 319-329.

ANDREWS, L. C., PHILLIPS, R. L. \& HOPEN, C. Y. (2001) Laser beam scintillation with applications, Bellingham, SPIE.

BATES, D. R. (1984) Rayleigh scattering by air. Planetary Space Science, 32, 785-790.

BLOOM, S., KOREVAAR, E., SCHUSTER, J. \& WILLEBRAND, H. (2003) Understanding the performance of free-space optics. Journal of Optical Networking, 2, 178-200.

GAGLIARDI, R. M. \& KARP, S. (1995) Optical Communications, New York, John Wiley.

GOODWIN, F. E. (1970) A review of operational laser communication systems. Proceedings of IEEE, 58, 1746-1752.

GOWAR, J. (1993) Optical communication systems, Prentice Hall.

GRABNER, M. \& KVICERA, V. (2009) Experimental study of atmospheric visibility and optical wave attenuation for free-space optics communications. http://ursifrance.institut-telecom.fr/pages/pages_ursi/URSIGA08/papers/F06p5.pdf. (Visited June 02 2009), Optical Crossing.

HEMMATI, H. (Ed.) (2006) Deep space optical communications, Canifornia, Wiley-Interscience 
JEGANATHAN, M. \& IONOV, P. Multi-gigabits-per-second optical wireless communications.

http://www.freespaceoptic.com/WhitePapers/Jeganathan\%20\%20(Optical\%20Cr ossing).pdf, (Visited May 20 2009), Optical Crossing.

KARP, S., GAGLIARDI, R. M., MORAN, S. E. \& STOTTS, L. B. (1988) Optical Channels: fibers, cluds, water and the atmosphere, New York, Plenum Press.

KAZAURA, K., OMAE, K., SUZUKI, T., MATSUMOTO, M., MUTAFUNGWA, E., MURAKAMI, T., TAKAHASHI, K., MATSUMOTO, H., WAKAMORI, K. \& ARIMOTO, Y. (2007) Performance evaluation of next generation free-space optical communication system. IEICE Transaction of Electronics, E90-C, 381-388.

KIASALEH, K. (2005) Performance of APD-based, PPM free-space optical communication systems in atmospheric turbulence. IEEE Transactions on Communications, 53, 14551461.

KILLINGER, D. (2002) Free space optics for laser communication through the air. Optics $\mathcal{E}$ Photonics News, 36-42.

KIM, I. I. \& KOREVAAR, E. (2001) Availability of free space optics and hybrid FSO/RF systems. Proceedings of SPIE: Optical wireless communications IV, 4530, 84-95.

OSCHE, G. R. (2002) Optical Detection Theory for Laser Applications, New Jersey, Wiley.

PELTON, J. N. (1998) Global satellite communications technology and systems. Baltimore, International Technology Research Institute, World Technology (WTEC) Division.

POPOOLA, W. O. \& GHASSEMLOOY, Z. (2009) BPSK subcarrier intensity modulated freespace optical communications in atmospheric turbulence. IEEE/OSA Journal of Lightwave Technology, , 27, 967-973.

POPOOLA, W. O., GHASSEMLOOY, Z., ALLEN, J. I. H., LEITGEB, E. \& GAO, S. (2008) Free-space optical communication employing subcarrier modulation and spatial diversity in atmospheric turbulence channel. IET Optoelectronic, 2, 16-23.

PRATT, W. K. (1969) Laser Communication Systems, New York, John Wiley \& Sons, Inc.

PROAKIS, J. G. (2004) Digital Communications, New York, McGraw-Hill.

ROCKWELL, D., A. \& MECHERLE, S., G. (2001) Optical wireless: Low-cost, broadband, optical accesss. www.freespaceoptic.com/WhitePapers/optical_wireless.pdf.

WILLEBRAND, H. \& GHUMAN, B. S. (2002) Free Space Optics: Enabling optical Connectivity in today's network, Indianapolis, IN, SAMS publishing. 


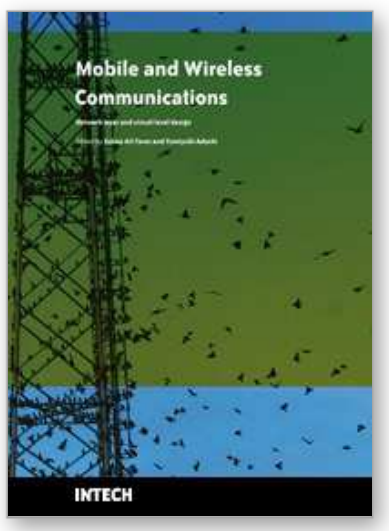

\author{
Mobile and Wireless Communications Network Layer and Circuit \\ Level Design \\ Edited by Salma Ait Fares and Fumiyuki Adachi
}

ISBN 978-953-307-042-1

Hard cover, 404 pages

Publisher InTech

Published online 01, January, 2010

Published in print edition January, 2010

Mobile and wireless communications applications have a clear impact on improving the humanity wellbeing. From cell phones to wireless internet to home and office devices, most of the applications are converted from wired into wireless communication. Smart and advanced wireless communication environments represent the future technology and evolutionary development step in homes, hospitals, industrial, vehicular and transportation systems. A very appealing research area in these environments has been the wireless ad hoc, sensor and mesh networks. These networks rely on ultra low powered processing nodes that sense surrounding environment temperature, pressure, humidity, motion or chemical hazards, etc. Moreover, the radio frequency $(\mathrm{RF})$ transceiver nodes of such networks require the design of transmitter and receiver equipped with high performance building blocks including antennas, power and low noise amplifiers, mixers and voltage controlled oscillators. Nowadays, the researchers are facing several challenges to design such building blocks while complying with ultra low power consumption, small area and high performance constraints. CMOS technology represents an excellent candidate to facilitate the integration of the whole transceiver on a single chip. However, several challenges have to be tackled while designing and using nanoscale CMOS technologies and require innovative idea from researchers and circuits designers. While major researchers and applications have been focusing on RF wireless communication, optical wireless communication based system has started to draw some attention from researchers for a terrestrial system as well as for aerial and satellite terminals. This renewed interested in optical wireless communications is driven by several advantages such as no licensing requirements policy, no RF radiation hazards, and no need to dig up roads besides its large bandwidth and low power consumption. This second part of the book, Mobile and Wireless Communications: Key Technologies and Future Applications, covers the recent development in ad hoc and sensor networks, the implementation of state of the art of wireless transceivers building blocks and recent development on optical wireless communication systems. We hope that this book will be useful for students, researchers and practitioners in their research studies.

\title{
How to reference
}

In order to correctly reference this scholarly work, feel free to copy and paste the following:

Ghassemlooy Z. and Popoola W. O. (2010). Terrestrial Free-Space Optical Communications, Mobile and Wireless Communications Network Layer and Circuit Level Design, Salma Ait Fares and Fumiyuki Adachi (Ed.), ISBN: 978-953-307-042-1, InTech, Available from: http://www.intechopen.com/books/mobile-andwireless-communications-network-layer-and-circuit-level-design/terrestrial-free-space-optical-communications

\section{INTECH}

open science | open minds 


\section{InTech Europe}

University Campus STeP Ri

Slavka Krautzeka 83/A

51000 Rijeka, Croatia

Phone: +385 (51) 770447

Fax: +385 (51) 686166

www.intechopen.com

\section{InTech China}

Unit 405, Office Block, Hotel Equatorial Shanghai

No.65, Yan An Road (West), Shanghai, 200040, China

中国上海市延安西路65号上海国际贵都大饭店办公楼 405 单元

Phone: +86-21-62489820

Fax: +86-21-62489821 
(C) 2010 The Author(s). Licensee IntechOpen. This chapter is distributed under the terms of the Creative Commons Attribution-NonCommercial-ShareAlike-3.0 License, which permits use, distribution and reproduction for non-commercial purposes, provided the original is properly cited and derivative works building on this content are distributed under the same license. 